\title{
Shear-band capturing using a multiscale extended digital image correlation technique
}

\author{
Julien Réthoré, François Hild, Stéphane Roux \\ Laboratoire de Mécanique et Technologie (LMT-Cachan) \\ Ecole Normale Supérieure de Cachan / UMR CNRS / Université Paris 6 \\ 61 Avenue du Président Wilson, F-94235 Cachan Cedex, France
}

\begin{abstract}
Finite elements have been used recently to solve the optical flow conservation principle invoked to determine displacement fields by digital image correlation. Inspired by these recent advances, and by the computational effort that has been accomplished during the past 10 years for the simulation of discontinuities by the eXtended Finite Element Method (XFEM), an extended correlation technique is introduced for capturing shear-band like discontinuities from images of real mechanical tests. Because of the specific information at hand (i.e., gray level images), this extended finite element approach is included in a non-linear multi-grid solver. The performances of the proposed approach are discussed on two examples, namely the analysis of a bolted assembly and the formation of Piobert-Lüders bands.
\end{abstract}

Key words: digital image correlation, extended finite element, multi-grid approach, shear-band, frictional interfaces 


\section{Introduction}

With the advances in digital imaging technology, larger and larger amount of data (i.e., pixels or voxels) are available, and are exploited to provide a rich and quantitative analysis of many phenomena. In particular, for solid mechanics applications, from strain gauge or extensometer measurements, one is often shifting to full field kinematic measurements using, say, digital image correlation (DIC). DIC is one out of several full field measurement techniques (see, e.g., (Coker and Filon, 1931; Guild, 1960; Dudderar and Simpkins, 1977)). As it simply consists in taking two images of the specimen surface (or volume) of interest in two different states, this technique has become very attractive. Furthermore, the number of images available may be used to compensate the bad quality or the poor resolution (for example by analyzing numerical movies obtained with a conventional camcorder (Bergonnier et al., 2003)).

Full field measurements become all the more interesting in identification procedures when the strain field to capture is heterogeneous. Many correlation algorithms rely on the local analysis of subsets subjected to different kinematics and gray level interpolations (Sutton et al., 1983, 2000). More recently, contin-

uous displacement field basis have been introduced in the general framework of maximization of the correlation between the reference and the displacementcorrected strained image such as B-splines (Cheng et al., 2002), polynomials (Schreier et al., 2002) or finite element interpolation functions (Sun et al., 2005). Alternative approaches based on a direct use of the optical flow conservation allow naturally for continuous displacement fields determination, as shown for Fourier series (Wagne et al., 2002), or finite element shape functions (Besnard et al., 2006). The finite element decomposition is particularly 
appealing because it allows one to bridge the gap between experiments and simulations since the kinematic bases may be identical in both cases. These approaches typically provide a better accuracy in the determination of the displacement field than local models, because at each pixel location, a faithful account of the displacement is provided.

However, one drawback of continuous bases is when localized phenomena arise such as cracks, shear-bands, sliding or frictional interfaces. They are smoothed out by the chosen basis, or may simply prevent the convergence of the procedure over the entire image when the displacement jump across the discontinuity is large. Alternative approaches require the partitioning of the domain into different parts that are further reassembled to estimate the discontinuity. Apart from the inconvenient nature of specific treatments to be designed for studied cases, the latter partitioning does not allow for partial coupling between different sub-domains, such as for instance the occurrence of tangent but no normal discontinuity. Following recent advances in computational mechanics, an eXtended digital image correlation (X-DIC) is introduced using the Partition of Unity Method (Babuska and Melenk, 1997) as in the eXtended Finite Element Method (Moës et al., 1999; Belytschko et al., 2001), the Generalized Finite Element Method (Strouboulis et al., 2000) or variants (Wells and Sluys, 2001; Wells et al., 2002).

A first publication by the authors on X-DIC was dedicated to analyzing cracks (Réthoré et al., 2007). The present paper is devoted to the study of discontinuities of the displacement component tangent to the discontinuity interface. A first application concerns interfaces between parts of an assembly. For large sliding, the elements cut by the discontinuity are very distorted and convergence within those elements is very difficult to achieve. To circumvent 
this aspect of the problem, a multi-grid algorithm coupled with the enriched interpolation is adopted in the present paper. Then, the technique is applied to shear-bands. The strain localization inside a shear-band has a typical extension that is given by an internal length scale (Wright, 2002). For the studied material, this scale is typically of the order of $10-100 \mu \mathrm{m}$ for structures whose scale is about $10^{-1}-10^{1} \mathrm{~m}$. Because of this large ratio between the internal length and the structure (or sample) size, multi-scale methods and/or adaptive remeshing are unavoidable for the simulation of shear-band formation. Measurement techniques are not as adaptive as numerical tools. Since multi-scale lenses or $10^{9}$-pixel sensors do not exist yet, the observation scale must be chosen a priori. At the structure scale at which the images are taken in this paper, the ratio between the internal length of the shear-band and the typical length of the specimen is about $10^{-3}$. For a $10^{6}$-pixel square image, the internal length is then of the order of 1 pixel. Consequently, the geometrical support of the strain localization zone is considered to be one dimensional (in a 2D formalism).

An application of the extended finite element method in conjunction with DIC focuses here on capturing experimentally tangential discontinuities. After a brief reminder of the image correlation principle (Section 2), the formulation of the optical flow conservation is applied in a finite element framework. Working with images implies the use of sophisticated solution strategies because of the high non-linearity of the problem and the presence of local minima. A non-linear multi-grid scheme is adopted and presented in Section 3. Then, the partition of unity property of finite element shape functions is invoked to enrich the interpolation basis of the displacement field with discontinuous functions. The enrichment proposed by Samaniego and Belytschko (2005) is followed. It consists in adding a discontinuous enrichment to the displacement 
interpolation but only along the tangential direction to the interface. Last, the two above mentioned applications are presented and the performance of the method is illustrated by an uncertainty analysis.

\section{Governing equations}

The principle of image correlation is derived from the "passive" advection of the local texture of two gray level images. The displacement field $\mathbf{u}$ between the reference image $f$ and the "deformed" image $g$ induced by this advection is to be estimated as accurately as possible. The local form of this principle reads

$$
g(\mathbf{x})=f(\mathbf{x}+\mathbf{u}(\mathbf{x}))
$$

This equation cannot be fulfilled pixelwise, and thus a weak form is generally formulated through the minimization of the global quantity

$$
\eta^{2}=\iint_{\Omega} \Phi^{2}(\mathbf{x}) \mathrm{d} \mathbf{x}
$$

where the local error density, $\Phi(\mathbf{x})$, is

$$
\Phi^{2}(\mathbf{x})=[f(\mathbf{x}+\mathbf{u}(\mathbf{x}))-g(\mathbf{x})]^{2}
$$

Then, a widely used approach is the maximization of a correlation coefficient (Sutton et al., 1983, 1986, 2000) by looking for, say, a piecewise constant displacement. Extension of this framework to more sophisticated continuous kinematic bases have been proposed (Cheng et al., 2002; Schreier et al., 2002). That route is not followed herein.

An alternative path (Roux et al., 2002; Wagne et al., 2002), not based on crosscorrelation maximization, consists in a direct minimization of the functional $\eta^{2}$ 
for arbitrary displacement fields. Therefore $\Phi$ is used as a local error indicator of the correlation procedure. This indicator gives a straightforward way of estimating the quality of the results.

\section{Resolution strategy}

Since the unknown displacement field $\mathbf{u}$ in the minimization problem appears as an argument of the gray level representation of the reference image $f$ [see Equation (2)], the problem is highly non-linear. Moreover, the texture of the images is itself irregular, it may lead to multiple local minima of $\eta$. For these reasons, an adapted solution strategy is to be used.

\subsection{Newton algorithm}

First, a Newton iterative procedure is elaborated to circumvent the non-linear aspects of the problem. Let us denote by $\mathbf{u}^{i}$ the approximation of the displacement field at iteration $i$. The problem is recast in the following form: find the displacement increment $\mathrm{d} \mathbf{u}=\mathbf{u}^{i+1}-\mathbf{u}^{i}$ minimizing $\eta$ such that

$$
\eta^{2}=\iint_{\Omega}\left[f\left(\mathbf{x}+\mathbf{u}_{i}+\mathrm{d} \mathbf{u}\right)-g(\mathbf{x})\right]^{2} \mathrm{~d} \mathbf{x}
$$

Assuming a small increment of the solution, the first term is linearized to provide

$$
\eta^{2}=\iint_{\Omega}\left[f\left(\mathbf{x}+\mathbf{u}^{i}\right)+\mathrm{d} \mathbf{u} \cdot \nabla f\left(\mathbf{x}+\mathbf{u}^{i}\right)-g(\mathbf{x})\right]^{2} \mathrm{~d} \mathbf{x}
$$


The right hand side is now expressed in an appropriate way

$$
\begin{aligned}
\eta^{2}= & \iint_{\Omega}\left[\mathrm{d} \mathbf{u} \cdot \nabla f\left(\mathbf{x}+\mathbf{u}^{i}\right)\right]^{2} \mathrm{~d} \mathbf{x} \\
& +2 \iint_{\Omega} \mathrm{d} \mathbf{u} \cdot \nabla f\left(\mathbf{x}+\mathbf{u}^{i}\right)\left[f\left(\mathbf{x}+\mathbf{u}^{i}\right)-g(\mathbf{x})\right] \mathrm{d} \mathbf{x} \\
& +\iint_{\Omega}\left[f\left(\mathbf{x}+\mathbf{u}^{i}\right)-g(\mathbf{x})\right]^{2} \mathrm{~d} \mathbf{x}
\end{aligned}
$$

As mentioned earlier, the displacement field is decomposed over a set of functions $\boldsymbol{\Psi}_{n}(\mathbf{x})$ (i.e., to be as general as possible, $\boldsymbol{\Psi}_{n}$ are vector functions that will be defined in the next Section). The approximation of the displacement field then reads

$$
\mathbf{u}(\mathbf{x})=\sum_{n \in \mathcal{N}} a_{n} \psi_{n}(\mathbf{x})
$$

where $\left(a_{n}\right)_{n \in \mathcal{N}}$ is the set of unknowns associated with the chosen interpolation functions ( $\mathcal{N}$ being a set of integers identifying the interpolation functions). By adopting matrix-vector notations, the minimization of Equation (6) is recast as a linear system

$$
\mathrm{M}^{i} \mathrm{~d} \mathbf{u}=\mathrm{b}^{i}
$$

where $\mathrm{M}^{i}$ is a "mass"-like matrix defined as

$$
M_{n m}^{i}=\iint_{\Omega}\left(\psi_{m} \cdot \nabla f\left(\mathbf{x}+\mathbf{u}^{i}\right)\right)\left(\psi_{n} \cdot \nabla f\left(\mathbf{x}+\mathbf{u}^{i}\right)\right) \mathrm{d} \mathbf{x}
$$

and $\mathrm{b}^{i}$ is the "force" vector

$$
b_{n}^{i}=\iint_{\Omega}\left(\psi_{n} \cdot \nabla f\left(\mathbf{x}+\mathbf{u}^{i}\right)\right)\left[g(\mathbf{x})-f\left(\mathbf{x}+\mathbf{u}^{i}\right)\right] \mathrm{d} \mathbf{x}
$$

The solution is then updated and the process is repeated until a convergence criterion associated with a given norm is reached. 


\subsection{Multi-grid solver}

Second, the solver must be able to avoid local minima. A multi-grid finite element strategy is utilized for this purpose. The region of interest of the reference image is decomposed into square elements $\Omega_{e}$ of pixels. The vertices of $\Omega_{e}$ are the nodes $\mathcal{N}_{e}$ that support finite element shape functions $\left(N_{j}\right)_{j \in \mathcal{N}_{e}}$.

Then, coarser grids are derived from the original one. At those grid levels, the elements consist of a set of "grains" (at the finest scale a grain is a pixel). The size of the grains is multiplied by a factor 2 in each direction from a certain grid level to the next coarser one. Using this notation, the element size in terms of "grains" is the same for all grids. This coarse graining strategy is useful because it allows for a regularization of the texture of the images. As noted above, original $f$ and $g$ pixel-wise functions are transformed into $f^{h}$ and $g^{h}$ grain-wise functions at the grid level $h$ ( $h$ being a power of 2 ). The values of $f^{h}$ and $g^{h}$ at a grid level are calculated as the average of $f^{h / 2}$ and $g^{h / 2}$ over the grains at level $h / 2$ contained in the grain at level $h$ (see Figure 1). This coarse graining step is viewed as a restriction operator

$$
f^{h}\left(\mathbf{x}_{G r}\right)=\mathrm{R}\left(f^{h / 2}, \mathbf{x}_{G r}\right)=\frac{1}{2^{2}} \sum_{g r \in G r} f^{h / 2}\left(\mathbf{x}_{g r}\right)
$$

where $\mathrm{R}$ is the restriction operator, $\sum_{g r \in G r}$ means a sum over the grains $g r$ at level $h / 2$ included in the grain $G r$ at level $h$. This regularization stage allows for filtering the short wavelengths of the texture at the coarser grids of the algorithm. These high frequency modes are then "grid" by "grid" reintroduced in the calculation. In practice, the size of the elements may be decreased to avoid too low numbers of elements for the coarser grids. Let us note that other procedures may have been chosen for regularization, such as Fourier filtering, but the very simple procedure proposed herein revealed 
efficient and satisfactory for the analyzed examples.

The prolongation of the displacement is performed using the bilinear interpolation of the finite element shape functions

$$
U_{h / 2, n}=\mathrm{P}\left(U_{h}, \mathbf{x}_{n}\right)=\sum_{j \in \mathcal{N}_{e}} U_{h, j} N_{j}\left(\mathbf{x}_{n}\right)
$$

where $\mathrm{P}$ is the prolongation operator, $U_{h / 2, n}$ is the displacement at node $n$ of level $h / 2,\left(\mathbf{x}_{n}\right)$ its position and $U_{h, j}$ the nodal values of the displacement at level $h$.

The initialization of the multi-grid solver uses a first rigid body approximation of the displacement over the entire region of interest by a Fast Fourier Transform (FFT) correlation algorithm (Hild et al., 2002). In the present case, there is no need for performing alternate cycles through different grid levels (so-called V or W cycles (Parsons and Hall, 1990)) in this application of a multi-grid solution strategy. Only one way down from the coarsest grid to the finest one is efficient enough to capture the global minimum. The algorithm is summarized in Figure 2.

\subsection{Special features}

Because the proposed algorithm is dedicated to image correlation, special fea-

tures are now discussed. A key point in reaching good convergence rates is the computation of $\nabla f\left(\mathbf{x}+\mathbf{u}^{i}\right)$ in the "mass" matrix involved in the linear system. The terms of the matrix are computed for each element and then assembled in the classical way for the finite element method. It is preferred to compute the gradient of the image $f$ in the frequency space using an FFT algorithm. For consistency, FFT is also used to translate the image for the sub-pixel part 
of $\mathbf{u}^{i}$. As a consequence, a mere constant approximation of $\mathbf{u}^{i}$ taken at the center of the element $\left(\mathbf{u}_{e}^{i}\right)$ is used in the computation of $f\left(\mathbf{x}+\mathbf{u}^{i}\right)$. Another consequence is that the displacement increment is performed with respect to $\mathbf{u}_{e}^{i}$. Thus, the system is rewritten in terms of $\mathbf{u}^{i+1}$ by shifting the contribution of $\mathbf{u}_{e}^{i}$ to the right hand side of Equation (8). If the element size is not a power of 2 grains, this strategy is used over a power of 2 grain size larger than the element size and the result is then restricted to the proper element zone. Furthermore, a symmetrization of the matrix is adopted by computing $\frac{1}{2} \nabla\left(f\left(\mathbf{x}+\mathbf{u}_{e}^{i}\right)+g(\mathbf{x})\right)$ instead of $\nabla f\left(\mathbf{x}+\mathbf{u}_{e}^{i}\right)$. This procedure is summarized in Figure 2.

The irregular texture of the images makes the usual Gauss quadrature inappropriate. Each above-defined "grain" carries an independent piece of information. The integration of $\mathrm{M}^{i}$ and $\mathrm{b}^{i}$ is consequently performed grain-wise. The convergence criterion is also an important feature of multi-grid approximation schemes. The criterion adopted here is based on the norm of the displacement increment, namely the mean value of the vector du. Working with images, the natural unit for the displacement is the pixel. This is equivalent to a normalization of the error criterion that is not carried out here. Typical values of the convergence to reach are about $10^{-3}$ to $10^{-4}$ pixel.

\section{Enriched displacement interpolation}

As mentioned in the Introduction Section, the phenomenon to capture is a discontinuity in the displacement field. Furthermore, the only component of the displacement tangential to the geometrical support of the discontinuity $\Gamma$ is assumed to suffer from a discontinuous behavior. Observing that finite 
element shape functions form a partition of unity

$$
\sum_{n \in \mathcal{N}} N_{n}(\mathbf{x})=1
$$

the discretization scheme in Equation (14) is an interpolating approximation for all functions $\zeta$ (Babuska and Melenk, 1997)

$$
\zeta_{\text {interp }}(\mathbf{x})=\sum_{n \in \mathcal{N}} \alpha_{n} N_{n}(\mathbf{x}) \zeta(\mathbf{x})
$$

If $\alpha_{n}=1$ for all $n$ then $\zeta_{\text {interp }}=\zeta$. For the applications discussed herein, $H_{n}(\mathbf{x}) \mathbf{t}_{\Gamma}$ is chosen as enrichment function for node $n$, where $\Gamma$ denotes the support of the discontinuity and $\mathbf{t}_{\Gamma}$ the unit vector tangent to this support. The domain $\Omega$ is separated by $\Gamma$ into two parts $\Omega^{-}$and $\Omega^{+}$(Figure 3 ), and $H_{n}$ is defined as

$$
H_{n}(\mathbf{x})=\mathcal{H}(\mathbf{x})-\mathcal{H}\left(\mathbf{x}_{n}\right)
$$

where $\mathcal{H}$ is the Heaviside step function whose value is 0 in $\Omega^{-}$and 1 in $\Omega^{+}$, and $\mathbf{x}_{n}$ gives the position of node $n$. This enrichment function, which was first introduced by Zi and Belytschko (2003), is preferred to the Heaviside or to the generalized Heaviside function because it is equal to 0 within the blending elements. Last, the discretization scheme reads

$$
\mathbf{u}(\mathbf{x})=\sum_{j=1,2} \sum_{n \in \mathcal{N}} a_{n j} N_{n}(\mathbf{x}) \mathbf{X}_{j}+\sum_{n \in \mathcal{N}_{\text {cut }}} d_{n} N_{n}(\mathbf{x}) H_{n}(\mathbf{x}) \mathbf{t}_{\Gamma}
$$

where $\left(N_{n}\right)_{n \in \mathcal{N}}$ are finite element shape functions, $\left(a_{n j}\right)_{n \in \mathcal{N}}$ degrees of freedom associated with those shape functions in each direction $\mathbf{X}_{1}$ and $\mathbf{X}_{2}$ of the image co-ordinate system, $\mathcal{N}_{\text {cut }}$ the set of nodes that hold an additional degree of freedom $d_{n}$ associated with the enrichment function $H_{n}(\mathbf{x}) \mathbf{t}_{\Gamma} \cdot \mathcal{N}_{c u t}$ is defined as the nodes of the elements cut by $\Gamma$. In those elements, since the displacement field is discontinuous, the matrix calculations are performed over two different parts $\Omega_{e}^{-}$and $\Omega_{e}^{+}$of the reference picture (Figure 4). Furthermore, 
if the displacement discontinuity is greater than 1 pixel, coarse graining of the reference image aggregates grains that are not neighbors in the deformed image. The proposed solution is to reconstruct an artificial reference image using the integer part of the mean value of a pre-estimate of the displacement on the + and - sides. The coarse graining stage thus becomes consistent with the kinematics.

\section{Shear interface detection}

The enriched kinematics adopted in the previous section requires the knowledge of the position of the discontinuity interface. It is proposed to proceed in two steps from a continuous displacement field (e.g., using P1 shape functions of 4-node finite elements as performed herein). First, find a relevant criterion for the detection of intense shear; second, select a straight line from the map of this criterion.

\subsection{Criterion}

Whatever the real displacement field within the elements cut by the interface (e.g., shear or sliding interface), a first guess using Q4P1 finite elements leads to high shear strains. For this reason, an equivalent strain in the spirit of Tresca (1864) is adopted

$$
\varepsilon_{e q}=\frac{\left|\varepsilon_{1}-\varepsilon_{2}\right|}{2}
$$

where $\varepsilon_{1}$ and $\varepsilon_{2}$ are the principal strains that are computed using the shape functions of the displacement interpolation. 


\subsection{Line selection}

The selection of a straight line from the map of $\varepsilon_{e q}$ is not always obvious. It is easier to select a spot in the co-ordinate system $(b, \theta)$ using a Radon transform, where the offset $b$ and the angle $\theta$ with respect to $\mathbf{X}_{1}$ are the natural quantities needed to describe the position and orientation of a straight line. The Radon transform (Barrett, 1984) of the field $\varepsilon_{e q}$ reads

$$
\mathcal{R}_{\varepsilon_{e q}}(b, \theta)=\iint \varepsilon_{e q}(x, y) \delta(y-\tan (\theta) x-b) \mathrm{d} x \mathrm{~d} y
$$

where $\delta$ is the Dirac delta function. Since straight lines are transformed into spots, it is easier to select $(b, \theta)$ maximizing $\mathcal{R}_{\varepsilon_{e q}}$. This property is illustrated by Figure 5 that shows a map for which the maximum is obtained along a line. The figure also depicts the Radon transform of this map and how it enables one to determine easily the corresponding line in the $(b, \theta)$ co-ordinate system.

In this paper, only straight interfaces are considered. It is not a limitation of the proposed extended digital image correlation technique but this simple geometry is rich enough for the considered applications. A shape optimization algorithm for cracks was proposed by the authors (Réthoré et al., 2007). It is applicable in the present enrichment framework if needed.

\section{Applications}

\subsection{Bolted assembly}

The first example deals with a bolted assembly of three plates made of an

aluminum alloy (Guinard et al., 2006). The center plate is pulled with respect 
to the left and right plates (Figure 6). To characterize globally friction between the plates, the displacement discontinuity is sought.

First, a study of the uncertainty of the displacement measurement is carried out. This is achieved by prescribing an artificial motion to the reference image. In the present case, the prescribed displacement exhibits a tangential discontinuity. The uncertainty $\sigma_{u}$ is estimated as

$$
\sigma_{u}=\left\langle\left\|\mathbf{u}-\mathbf{u}_{p}\right\|^{2}\right\rangle^{1 / 2}
$$

where $\mathbf{u}_{p}$ is the prescribed displacement field and $\langle v\rangle$ denotes the average value of $v$ over the entire region of interest. The standard uncertainty $\sigma_{u}$ is calculated for different mesh sizes varying from 8 to 64 pixels and different prescribed displacement values in the $[0,1]$-pixel range. The average of uncertainty for the different displacement values is plotted as a function of the element size. Figure 7 shows the results for the entire displacement map and Figure 8 those for the displacement jump. As anticipated, a standard finite element approximation leads to an unsatisfactory behavior, namely, the uncertainty increases as the element size increases. This is due to the bias introduced by the projection of a discontinuous field onto a standard continuous finite element basis (see Figure 7, Q4P1 Projection). The prescribed motion is not in the space described by Q4P1 shape functions. The uncertainty is mainly due to projection errors. Consequently, a numerical-type of convergence is retrieved i.e., the uncertainty increases with the element size. On the contrary, when an appropriate kinematics is introduced in the discretization basis, the uncertainty decreases as the mesh size increases. For this example, the level of uncertainty is as low as $6 \times 10^{-4}$ pixel for 64-pixel elements. Similar values are obtained for the uncertainty associated with the displacement jump. These (very low) values are obtained because the deformed picture is created artificially. This 
procedure allows one to evaluate the performance of the correlation algorithm when dealing with actual reference pictures and given displacement fields. It is worth noting that considering normal discontinuous enrichment functions does not lead to higher uncertainty levels on the displacement jump determination.

In practice, a region of interest is selected (Figure 6). A sliding interface is supposed to be detected within this zone. A continuous approximation of the vertical displacement field (Figure 9-b) exhibits elements with high levels of shear strain. This is confirmed by Figure 10. In this figure, one observes the equivalent Tresca strain map as well as its Radon transform. As expected from Section 5, the Radon transform provides an easy selection of the line with the highest equivalent strain. Using this line as the geometrical support of the discontinuity, the displacement approximation basis is now enriched with the discontinuous functions $H_{n} \mathbf{t}_{\Gamma}$. The results are shown in Figure 9-c. When compared with the case where no enrichment is used (Figure 9-b), one notes that it avoids smearing the discontinuity over the elements cut by the discontinuity.

Figures 9-a, 11-a and 12-a also show the results obtained with only 1 grid of 32-pixel elements (depicted in Figure 6). They are quite different from those obtained by using 5 grids with 32-grain elements (the finest one being the mesh depicted in Figure 6). One observes in Figure 12-a that the residual error is really higher over the right part of the region of interest. This is emphasized by Figure 13 where the convergence criterion is plotted as a function of the number of Newton iterations from the coarsest grid on. When using only 1 grid, convergence is not obtained, whereas the use of 5 grids enables the analysis to converge. However, the convergence criterion (here $10^{-4}$ pixel) is not reached when using a Q4P1 interpolation scheme. The enrichment procedure (used 
from grid 3 on) allows for a better convergence as illustrated by Figure 14. Fewer iterations are needed with the enriched kinematics, especially for the finer scales where most of the computation time is needed.

Table 1 lists the CPU time spent in the different grid levels. Because a better convergence is obtained with the enrichment, less time is spent in the finest grids that are more time consuming. Last, the CPU time is divided by a factor of 2.36, even though the analysis was stopped after 20 iterations on the finest grid. As the convergence criterion is not reached with the standard interpolation, the actual ratio is higher. The convergence error is illustrated by Figure 15. It is observed, as in Figure 12, that the mean error level is lower when several grid levels are used to perform the analysis, and even lower when discontinuous functions are introduced in the interpolation basis. Furthermore, the enrichment procedure allows one to coarsen the mesh, and thus decrease the measurement uncertainty (Figures 7 and 8), yet capture very accurately the discontinuities and the surrounding displacements. This result is shown in Figure 16 for the whole displacement map, and in Figure 17 for the displacement jump along the discontinuity line. The mean displacement discontinuity is about 7.5 pixels in this case, and explains why convergence is more difficult to obtain with a standard interpolation scheme and even more if only on grid is used.

Last, the error map $\Phi$ (Figure 12) enables one to compare the quality of the results obtained with different interpolation bases. The dynamic range of the picture is equal to 256 gray levels. The improvement obtained with the proposed extended image correlation algorithm is clearly visible in this figure. Using standard finite elements (Q4P1), high error levels are observed within the entire zone defined by the elements cut by the interface. Using an enriched 
Table 1

Comparison of the CPU time in seconds for Q4 and extended correlation analyses of the bolted assembly.

\begin{tabular}{|l||c|c|c|c|c||c|}
\hline Analysis & Grid 1 & Grid 2 & Grid 4 & Grid 8 & Grid 16 & Total \\
\hline Q4 5 grids & 0.5 & 2.7 & 8.9 & 10.9 & 61.5 & 84.5 \\
\hline X-Q4 5 grids & 0.5 & 2.7 & 5.0 & 6.0 & 21.5 & 35.7 \\
\hline Ratio & 1.0 & 1.0 & 1.78 & 1.81 & 2.86 & 2.36 \\
\hline
\end{tabular}

kinematic basis (X-Q4), the high error zone reduces to the interface itself. The mean error in a band of width equal to two elements around the interface is 22.1 gray levels with standard P1 shape functions. The mean error decreases to 4.2 gray levels when an enriched interpolation basis is used. Part of this residual is due to a slight misalignment of the discontinuity line.

\subsection{Piobert-Lüders band}

The second example focuses on the analysis of Piobert-Lüders bands during a tension test controlled by using digital image correlation (Fayolle et al., 2007) instead of a strain gauge or an extensometer. The tested steel exhibits an elastic perfectly plastic behavior prone to localized shear bands. Outside the band, the material is in the elastic regime, and the strains are much smaller than the plastic strains in the band. However, no specific assumption concerning this feature is postulated in the present approach. Part of the experimental setup is shown in Figure 18. The quality of the images is poorer than for the previous example (i.e., the local fluctuations are much smaller), thus higher uncertainty levels (Figures 19 and 20) are observed. This result shows that 
the quality of the texture (i.e., its gradient as shown by the linearized functional (2) to minimize) is crucial when high performances of the correlation procedure are needed.

The region of interest is the upper right corner of the specimen where localization occurs (Figures 21-a and 22-a). Figure 23 shows the equivalent strain map and its Radon transform. The strain localization line appears clearly on those pictures. It therefore enables for the detection of the position of the interface supporting the kinematic discontinuity. In the present case, the geometry of the sample cannot be used to locate the discontinuity line as in the previous example. One may also note that at the scale of the picture (the physical size of 1 pixel is $86 \mu \mathrm{m})$, the shear-band appears as a line. If a smaller scale had been reached by the observation setup, a finite band width may have been revealed. The displacement maps tangential to the detected interface are shown in Figure 24. Using the proposed extended digital image correlation technique, strain localization is captured in the detected interface whereas Q4P1 digital image correlation only provides a smoothed description of this phenomenon. If a more accurate description of the localization interface were required with Q4P1 finite elements, smaller elements should have been used, yet leading to higher uncertainty levels (see Figure 19). Hence, one of the major advantages of using extended finite element in the context of digital image correlation is that it allows for the description of localized phenomena with coarser meshes i.e., with lower uncertainty levels.

From the uncertainty analysis, it is concluded that the measured normal displacement jump (Figure 25-c) when the corresponding enrichment is included in the approximation, is lying within the uncertainty range. These results are very close to those obtained when the normal enrichment is discarded (Fig- 
ure 25-b) or when a continuous field is sought (Figure 25-a). One observes in Figure 26 that the mean value of the normal discontinuity is about 0.1 pixel for an uncertainty level of 0.103 pixel. Concerning the tangential discontinuity, the results obtained with a tangential enrichment only and a full enrichment are very similar, the average value being 0.9 pixel Figure 24. Let us note that even if the shear-band is not expected to display a discontinuity in the normal direction in three dimensions, the fact that only a face view is examined may induce an apparent normal discontinuity in the plane. The latter would be expected ideally to be the projection of the 3D tangential discontinuity onto the observation plane.

The error maps are shown in Figure 27. Since the displacement discontinuity is less than 1 pixel, the difference between a standard and enriched approximation is less perceptible than in the previous example. However, the mean error within the elements cut by the shear-band decreases from 422 gray levels to 355 gray levels (the dynamic range of the images is 65536 gray levels). This last result is the only evidence given here to validate the measured displacement field.

\section{Summary}

Written within a finite element framework, image correlation procedures lead to the minimization of a non-linear functional. Since image textures are highly irregular, trapping into local minima of this functional must be avoided. For this purpose, a Newton iterative procedure coupled with a multi-grid solver is a reliable solution strategy. Discontinuous functions are introduced in the displacement interpolation by exploiting the partition of unity property of 
finite element shape functions. This discontinuous enrichment chosen herein only concerns the component of the displacement tangent to the geometrical support of the discontinuity.

The Radon transform is then used to determine the geometry of the discontinuity line from the map of equivalent strain. As shear is preponderant in the formation of Piobert-Lüders band or in the kinematics of frictional interfaces, the equivalent "Tresca" strain is used for the detection of the interface. The proposed examples illustrate the performance of the approach and an uncertainty analysis allows one to show that the enrichment for the normal displacement discontinuities can be discarded.

When studying localized phenomena, the localization band is usually on a region of the sample of dimension $n-1$ (e.g., 1 in $2 \mathrm{D}$ situations) whereas the rest of the sample remains less active. Consequently, the extended digital image correlation technique proposed herein becomes all the more attractive since it allows for the description of localized phenomena with coarser meshes i.e., with lower uncertainty levels in the estimation of the continuous and the discontinuous components of the displacement.

The approach proposed herein may be seen as a mathematically appropriate generalization of a procedure investigators are used to. It consists in treating the above and below side of the image separately after a first estimation of the displacement field over the entire image. As a limit to this practical procedure, the extended finite element interpolation basis used herein gives access to the displacement discontinuity after the interface has been detected using an appropriate criterion and its Radon transform. Another aspect of the present approach is that the obtained displacement invokes the same discretization basis as one may use for the numerical simulation proposed, for example, 
by Samaniego and Belytschko (2005).

Last, dealing with more complex interface geometries is not a limitation of the extended digital image correlation technique discussed herein. The main issue concerns the determination of the geometrical support of the band which may be as irregular as required (Réthoré et al., 2007). However, shear-bands are usually not geometrically as irregular as cracks and the present approximation of their geometry using straight lines remains reliable at the scale of observation.

\section{Acknowledgments}

This work is part of a project (PHOTOFIT) funded by the Agence Nationale de la Recherche. The authors wish to thank Stéphane Guinard, Nicolas Swiergiel and Julien Vignot of EADS for providing the pictures of the bolted assembly analyzed herein. 


\section{References}

Babuska, I., Melenk, J., 1997. The partition of unity method. International Journal for Numerical Methods in Engineering 40, 727-758.

Barrett, H., 1984. The Radon transform and its applications. Progress in optics $21,217-286$.

Belytschko, T., Moës, N., Usui, S., Parimi, C., 2001. Arbitrary discontinuities in finite elements. International Journal for Numerical Methods in Engineering 50 (4), 993-1013.

Bergonnier, S., Hild, F., Roux, S., 2003. Analyse d'une cinématique hétérogène. Revue des Composites et des Matériaux Avancés 13 (3), 293302.

Besnard, G., Hild, F., Roux, S., 2006. 'finite-element' displacement fields analysis from digital images: Application to portevin-le châtelier bands. Experimental Mechanics 46, 789-803.

Cheng, P., Sutton, M. A., Schreier, H. W., McNeill, S. R., 2002. Full-field speckle pattern image correlation with B-spline deformation function. Experimental Mechanics 42 (3), 344-352.

Coker, E., Filon, L., 1931. A treatise on Photoelasticity. Cambridge University Press, New York.

Dudderar, T., Simpkins, P., 1977. Laser speckle photography in a fluid medium. Nature 270, 45-47.

Fayolle, X., Calloch, S., Hild, F., 2006. Controlling testing machines with digital image correlation. Experimental Techniques, 31, 57-63.

Guild, J., 1960. Diffraction gratins as measuring scales, practical guide to metrological use of Moiré fringes. Oxford University Press, Oxford.

Guinard, S., Swiergiel, N., Vignot, J., 2006. Personal communication. 
Hild, F., Raka, B., Baudequin, M., Roux, S., Cantelaube, F., 2002. Multiscale displacement field measurements of compressed mineral wool samples by digital image correlation. Applied Optics 41 (32), 6815-6828.

Moës, N., Dolbow, J., Belytschko, T., 1999. A finite element method for crack growth without remeshing. International Journal for Numerical Methods in Engineering 46 (1), 133-150.

Parsons, ID., Hall, JF., 1990. The multigrid method in solid mechanics: part I-algorithm description and behaviour, International Journal for Numerical Methods in Engineering 29, 719-737.

Réthoré, J., Hild, F., Roux, S., 2006. Extended digital image correlation with crack shape optimization. International Journal for Numerical Methods in Engineering, in press (DOI: 10.1002/nme.2070).

Roux, S., Hild, F., Berthaud, Y., 2002. Correlation image velocimetry: A spectral approach. Applied Optics 41 (1), 108-115.

Samaniego, E., Belytschko, T., 2005. Continuum-discontinuum modelling of shear bands. International Journal for Numerical Methods in Engineering $62(13), 1857-1872$.

Schreier, H. W., Sutton, M. A., 2002. Systematic Errors in Digital Image Correlation Due to Undermatched Subset Shape Functions. Exp. Mechanics, Vol. 42 (3), 303-310.

Strouboulis, T., Babuska, I., Copps, K., 2000. The design and analysis of the generalized finite element method. Computer Methods in Applied Mechanics and Engineering 181, 43-69.

Sun, Y., Pang, J., Wong, C., Su, F., 2005. Finite-element formulation for a digital image correlation method. Applied Optics 44 (34), 7357-7363.

Sutton, M., Cheng, M., Peters, W., Chao, Y., McNeill, S., august 1986. Application of an optimized digital image correlation method to planar defor- 
mation analysis. Image Vision Computing 4 (3), 143-150.

Sutton, M., McNeill, S., Helm, J., Chao, Y., 2000. Photomechanics. Springer, Ch. Advances in two-dimensional and three-dimensional computer vision, pp. 323-372.

Sutton, M., Wolters, W., Peters, W., Ranson, W., McNeill, S., 1983. Determination of displacements using an improved digital correlation method. Image Vision Computing 1 (3), 133-139.

Tresca, H., 1864. Mémoire sur l'écoulement des corps solides soumis à de fortes pression. Comptes Rendus de l'Académie des Sciences 59, 754-758.

Wagne, B., Roux, S., Hild, F., 2002. Spectral Approach to Displacement Evaluation From Image Analysis, European Physical Journal AP 17, 247-252.

Wells, G., Sluys, L., 2001. A new method for modeling cohesive cracks using finite elements. International Journal for Numerical Methods in Engineering $50,2667-2682$.

Wells, G., Sluys, L., de Borst, R., 2002. Simulating the propagation of displacement discontinuities in a regularized strain-softening medium. International Journal for Numerical Methods in Engineering 53, 1235-1256.

Wright, T., 2002. The Physics and Mathematics of Adiabatic Shear Bands. Cambridge University Press.

Zi, G., Belytschko, T., 2003. New crack-tip elements for xfem and applications to cohesive cracks. International Journal for Numerical Methods in Engineering 57 (15), 2221-2240. 


\section{List of Figures}

1 Depiction of the coarse graining step for three grid levels.

2 Multi-grid resolution strategy. The maximum number of iterations for one grid level and the user defined convergence criterion are adapted to the grid level (typically multiplied by $h)$. The ratio between the coarsest grid and the finest is also a user defined variable (a standard value is 4 ).

3 The domain of interest $\Omega$ is cut by a discontinuity interface $\Gamma$ into two parts $\Omega^{+}$and $\Omega^{-}$. Circles depict the nodes that hold enriched degrees of freedom.

4 Typical integration configuration: over the initial image no specific treatment is needed whereas the integration over the deformed within the elements cut by the discontinuity requires two sub-domains.

5 Left: example map; right: Radon transform of the map.

6 Region of interest, mesh (grid 1) and detected interface for the studied assembly

$7 \quad$ Uncertainty of the displacement field for an artificial discontinuous displacement in the tangential direction.

8 Uncertainty of the displacement jump for an artificial discontinuous displacement in the tangential direction. 
$9 \quad$ Vertical displacement map (in pixels) using 32-pixel elements with (a) Q4 interpolation on 1 grid,(b) Q4 interpolation on 5 grids, (c) discontinuous enrichment tangent to the interface on 5 grids

10 Left: criterion map for 16-pixel elements; right: Radon transform of the criterion

11 Horizontal displacement map (in pixels) using 32-pixel elements with (a) Q4 interpolation on 1 grid,(b) Q4 interpolation on 5 grids, (c) discontinuous enrichment tangent to the interface on 5 grids

12 Error map (in pixels) using 32-pixel elements with (a) Q4 interpolation on 1 grid,(b) Q4 interpolation on 5 grids, (c) discontinuous enrichment tangent to the interface on 5 grids

13 Comparison of the convergence for analyses using 1 or 5 grids of 32-grain elements with a Q4P1 interpolation. The convergence criterion to reach is $10^{-4}$ pixel. Neither 1 -grid nor 5-grid analyses allow for convergence at the true scale (grid 1)

14 Comparison of the convergence for analyses using 5 grids of 32-grain elements with a Q4P1 interpolation or an enrichment with discontinuous functions (the enrichment is introduced at grid 3). The convergence criterion to reach is $10^{-4}$ pixel. 
15 Comparison of the mean error for analyses using 1 grid with a Q4 interpolation, 5 grids with a Q4 interpolation and 5 grids with an enrichment with discontinuous functions (the enrichment is introduced at grid 3). 32-grain elements are used for the analyses.

16 Vertical displacement map (in pixels) using tangential enrichment for different discretizations: (a) 8-pixel elements, (b) 16-pixel elements, (c) 32-pixel elements, (d) 48-pixel elements

17 Comparison of displacement jump profiles for different element sizes

18 Region of interest, mesh (grid 1) and detected shear band

19 Uncertainty of the displacement map for an artificial discontinuous displacement in the tangential direction.

20 Uncertainty of the displacement jump for an artificial discontinuous displacement in the tangential direction.

21 Vertical displacement map (in pixels) using 32-pixel elements with (a) Q4 interpolation, (b) discontinuous enrichment tangent to the band, (c) discontinuous enrichment tangent and normal to the band

22 Horizontal displacement map (in pixels) using 32-pixel elements with (a) Q4 interpolation, (b) discontinuous enrichment tangent to the band, (c) discontinuous enrichment tangent and normal to the band 
23 Top: equivalent strain map; bottom: detection criterion

24 Tangent displacement map (in pixels) using 32-pixel elements with (a) Q4 interpolation, (b) discontinuous enrichment tangent to the band, (c) discontinuous enrichment tangent and normal to the band

25 Normal displacement map (in pixels) using 32-pixel elements with (a) Q4 interpolation, (b) discontinuous enrichment tangent to the band, (c) discontinuous enrichment tangent and normal to the band

26 Displacement jumps for 32-pixel elements with or without normal enrichment

27 Error map (in gray levels) using 32-pixel elements with (a) Q4 interpolation, (b) discontinuous enrichment tangent to the band, (c) discontinuous enrichment tangent and normal to the band. The scale is saturated to 5000 gray levels in an initial range of $2^{16}$ gray levels 


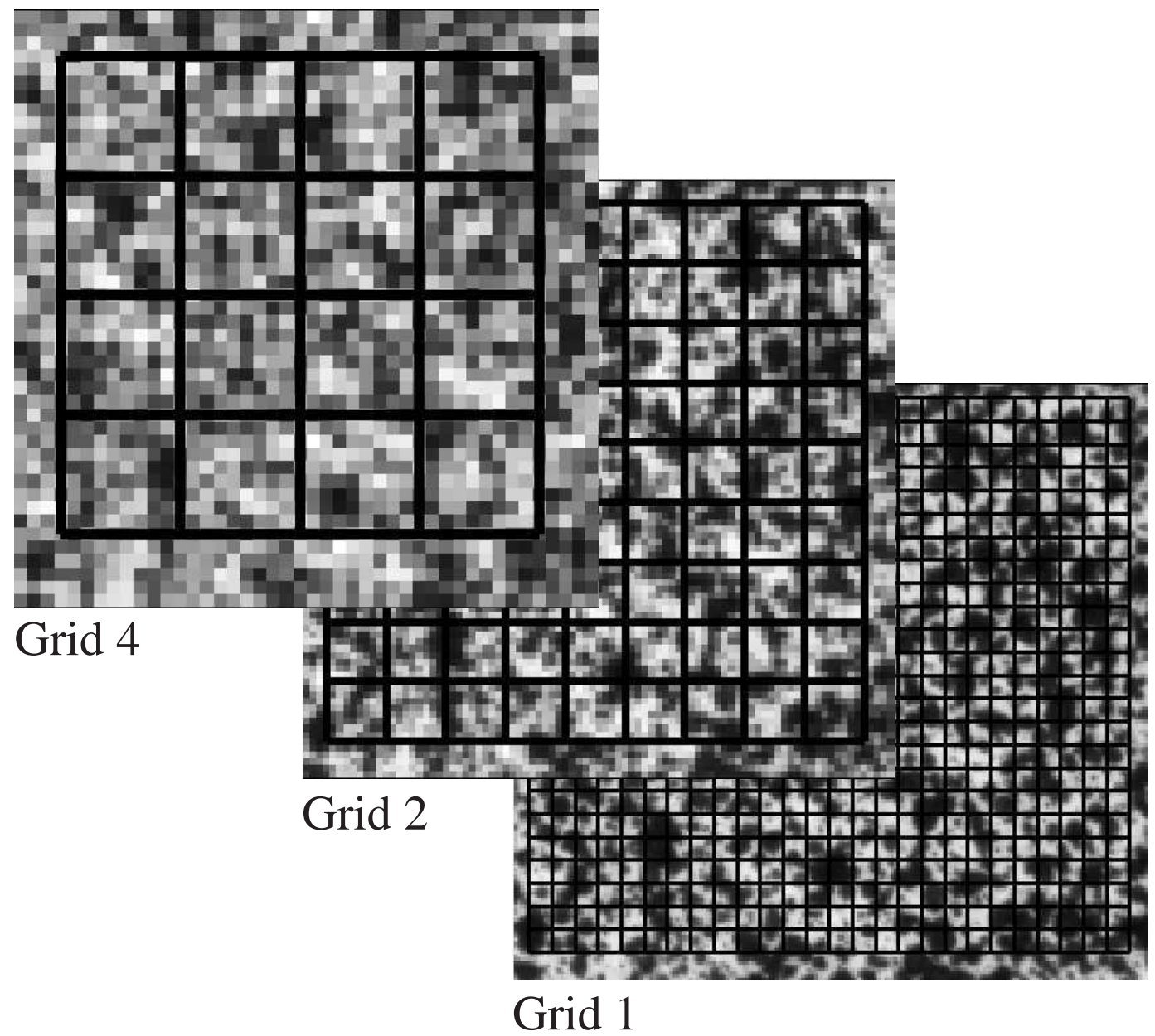

Fig. 1. Depiction of the coarse graining step for three grid levels. 
first estimation of rigid body translation by FFT

for h from $2^{\text {coarse }}$ to $2^{\text {fine }}$ do

coarse graining: $f^{h}, g^{h}$

prolongation of the displacement, set in $\mathbf{u}^{0}$

while $i \leq i_{\max }(h)$ or $E r \geq \varepsilon * h$ do

loop

compute the displacement at the center of element $e: \mathbf{u}_{e}^{i}$

index shifting of $f$ by the integer part of $\mathbf{u}_{e}^{i}$

translation of $f$ by the sub-pixel part of $\mathbf{u}_{e}^{i}$ using FFT

computation by FFT of $\frac{1}{2} \nabla\left(f\left(\mathbf{x}+\mathbf{u}_{e}^{i}\right)+g(\mathbf{x})\right)$

computation of $M_{n m}^{i}$

computation of $b_{n}^{i}$

accounting for $\mathbf{u}_{e}^{i}: b_{n}^{i}=b_{n}^{i}+\sum_{m} M_{n m}^{i} \mathbf{u}_{e}^{i}$

assembly

end loop

solve $\mathrm{M}^{i} U^{i+1}=\mathrm{b}^{i}$

compute error $\mathrm{Er}$

end while

end for

Fig. 2. Multi-grid resolution strategy. The maximum number of iterations for one grid level and the user defined convergence criterion are adapted to the grid level (typically multiplied by $h$ ). The ratio between the coarsest grid and the finest is also a user defined variable (a standard value is 4 ). 


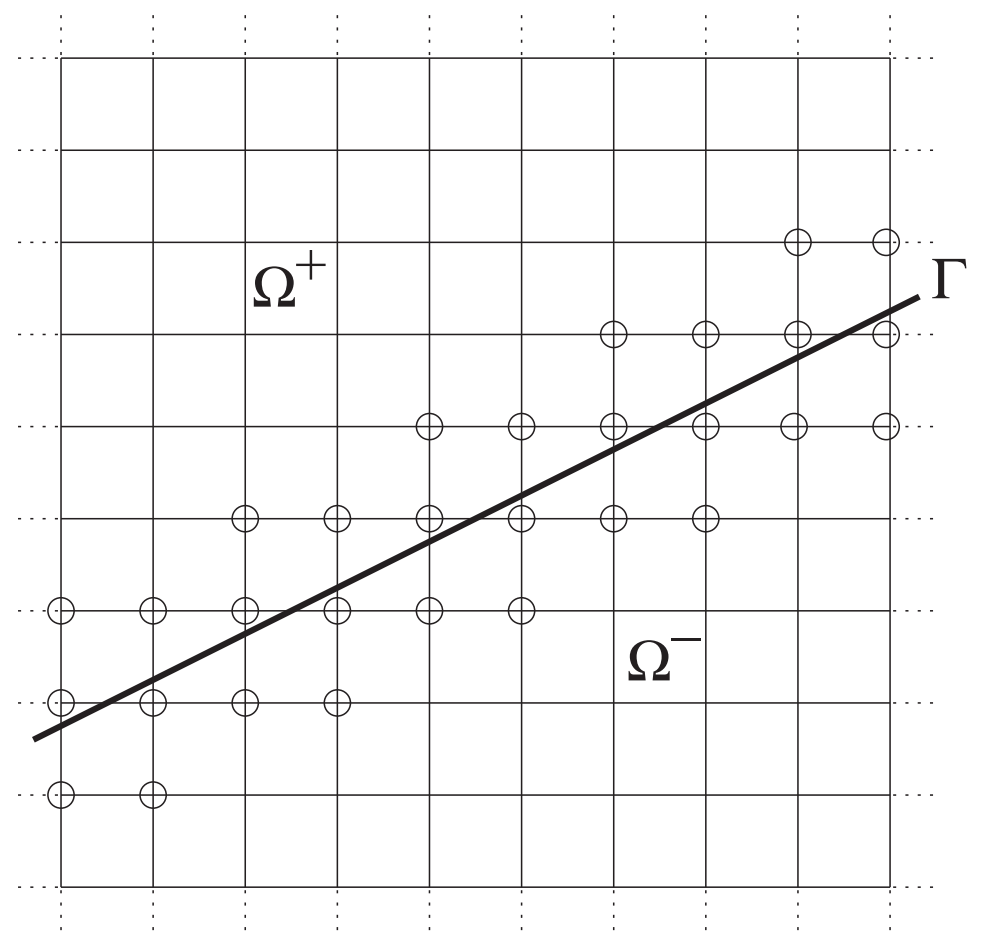

Fig. 3. The domain of interest $\Omega$ is cut by a discontinuity interface $\Gamma$ into two parts $\Omega^{+}$and $\Omega^{-}$. Circles depict the nodes that hold enriched degrees of freedom.

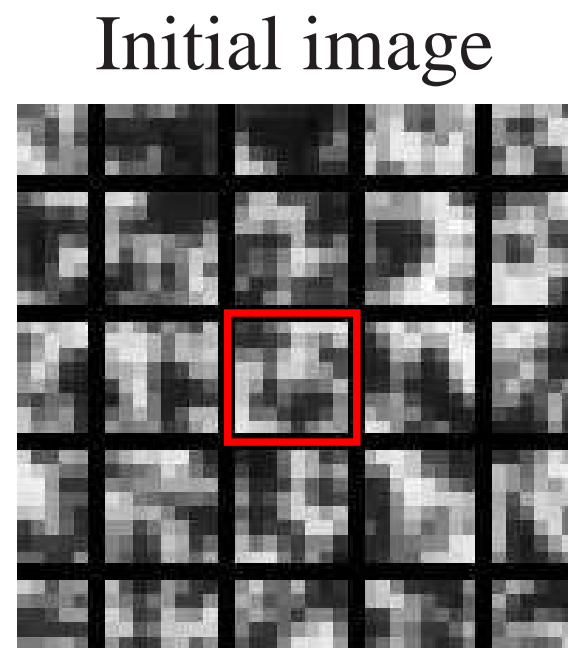

\section{Deformed image}

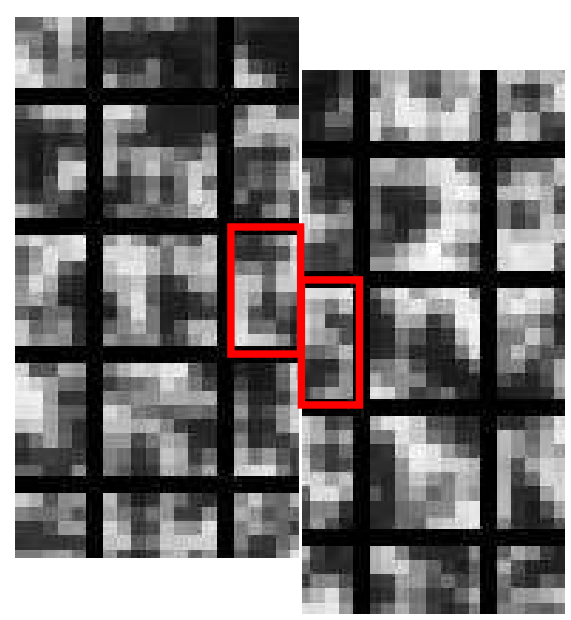

Fig. 4. Typical integration configuration: over the initial image no specific treatment is needed whereas the integration over the deformed within the elements cut by the discontinuity requires two sub-domains. 

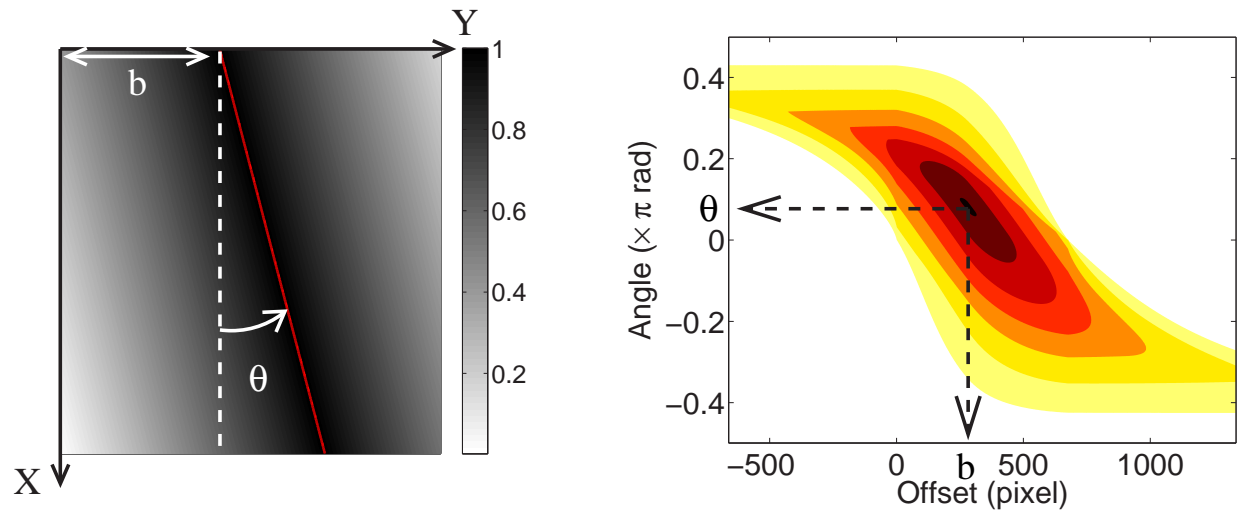

Fig. 5. Left: example map; right: Radon transform of the map. 


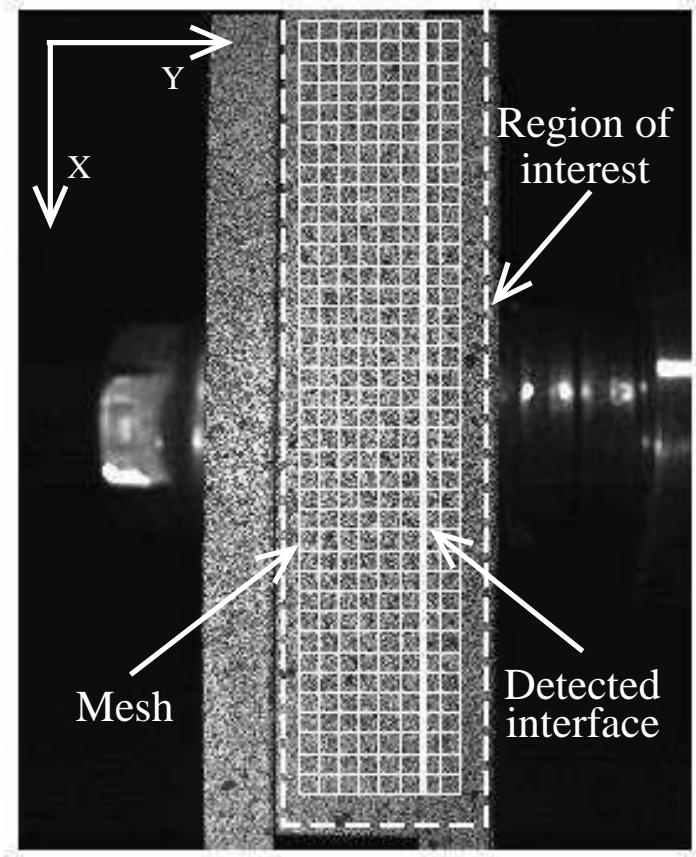

Fig. 6. Region of interest, mesh (grid 1) and detected interface for the studied assembly

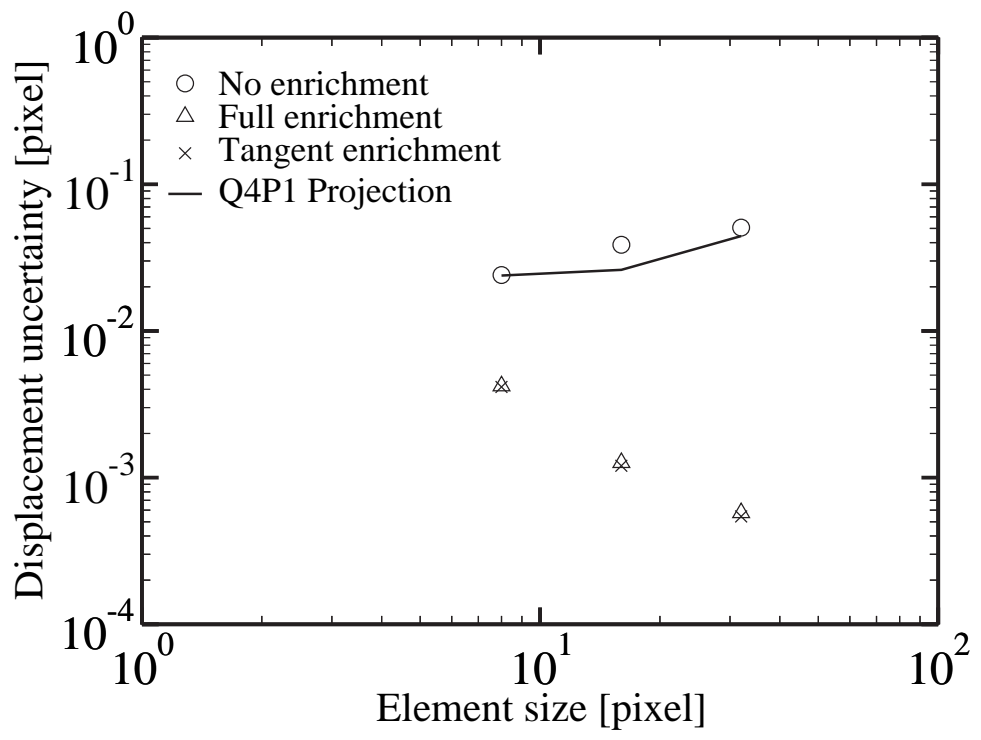

Fig. 7. Uncertainty of the displacement field for an artificial discontinuous displacement in the tangential direction. 


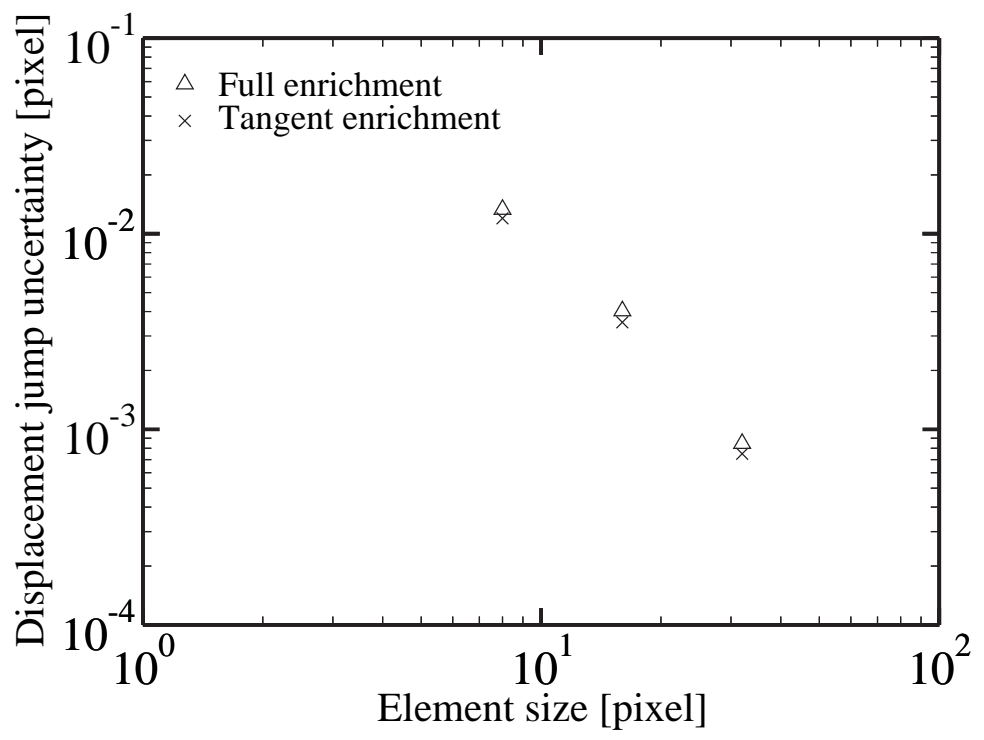

Fig. 8. Uncertainty of the displacement jump for an artificial discontinuous displacement in the tangential direction. 


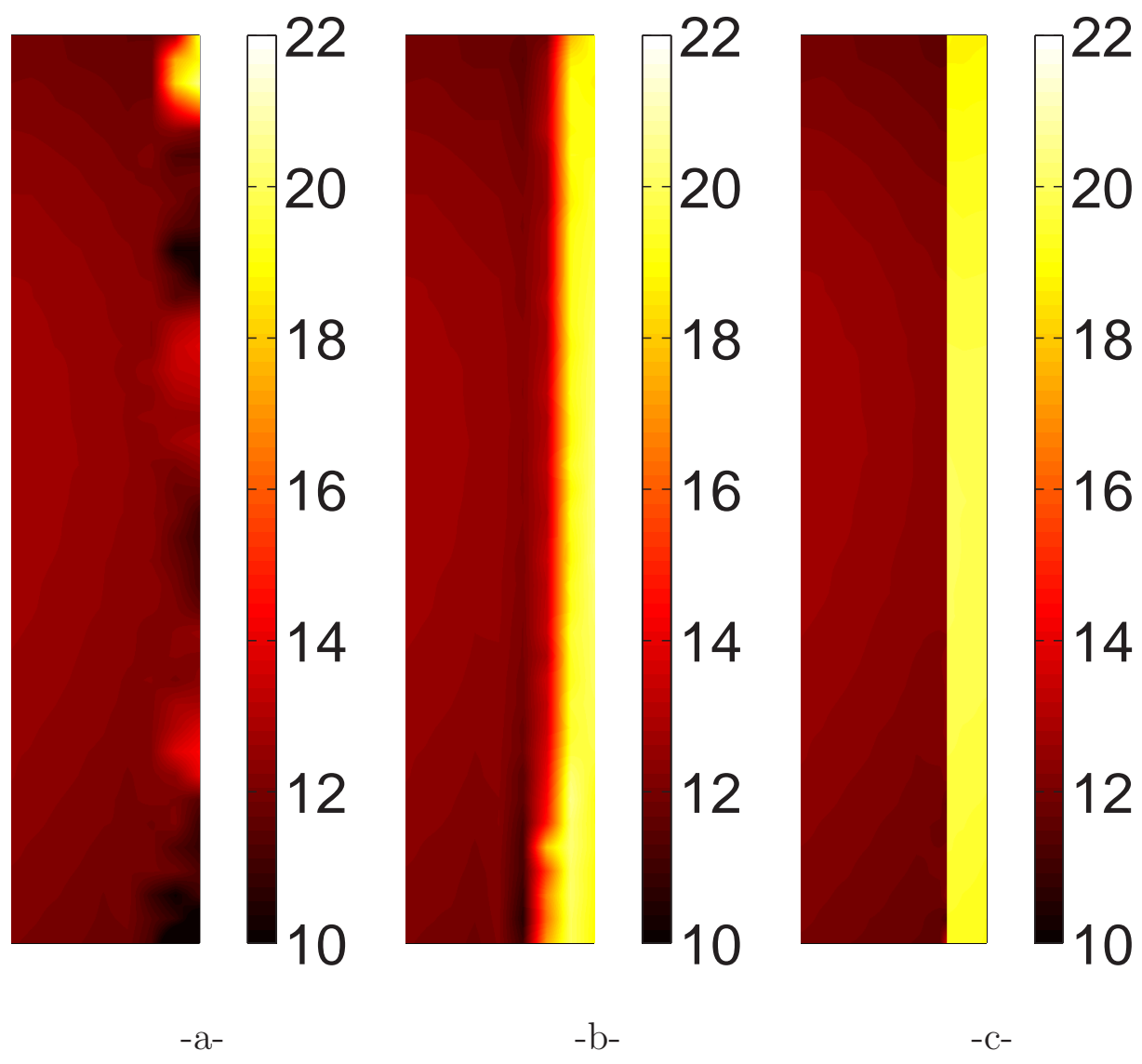

Fig. 9. Vertical displacement map (in pixels) using 32-pixel elements with (a) Q4 interpolation on 1 grid,(b) Q4 interpolation on 5 grids, (c) discontinuous enrichment tangent to the interface on 5 grids 


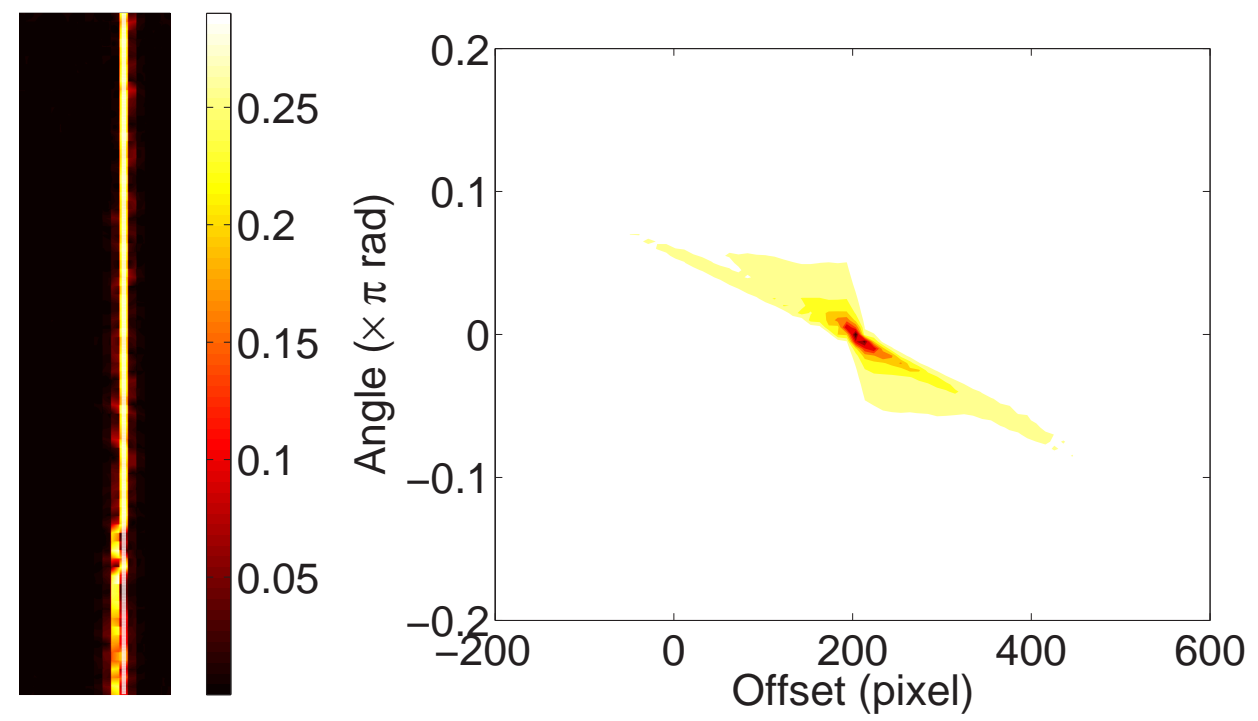

Fig. 10. Left: criterion map for 16-pixel elements; right: Radon transform of the criterion 


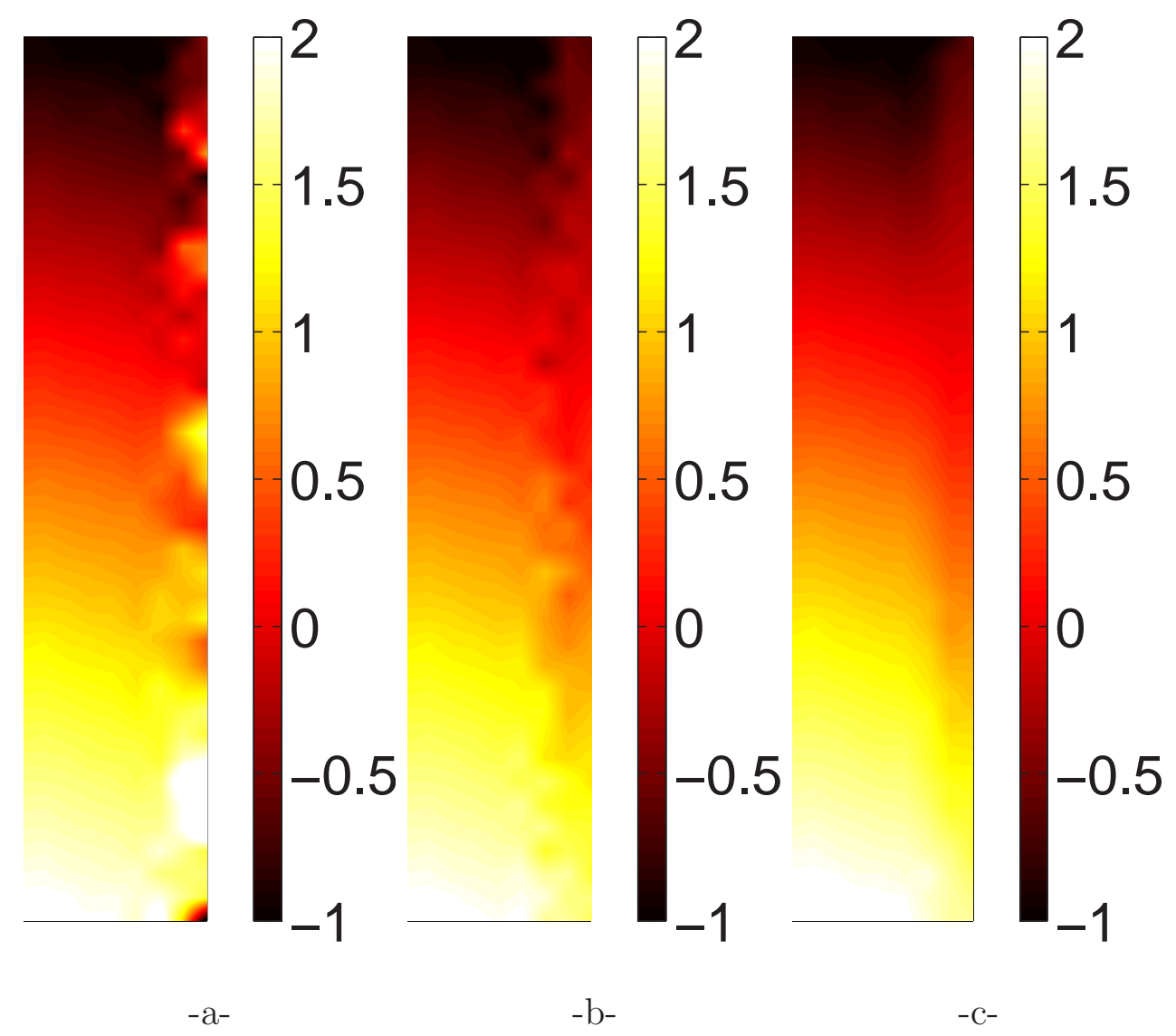

Fig. 11. Horizontal displacement map (in pixels) using 32-pixel elements with (a) Q4 interpolation on 1 grid,(b) Q4 interpolation on 5 grids, (c) discontinuous enrichment tangent to the interface on 5 grids 


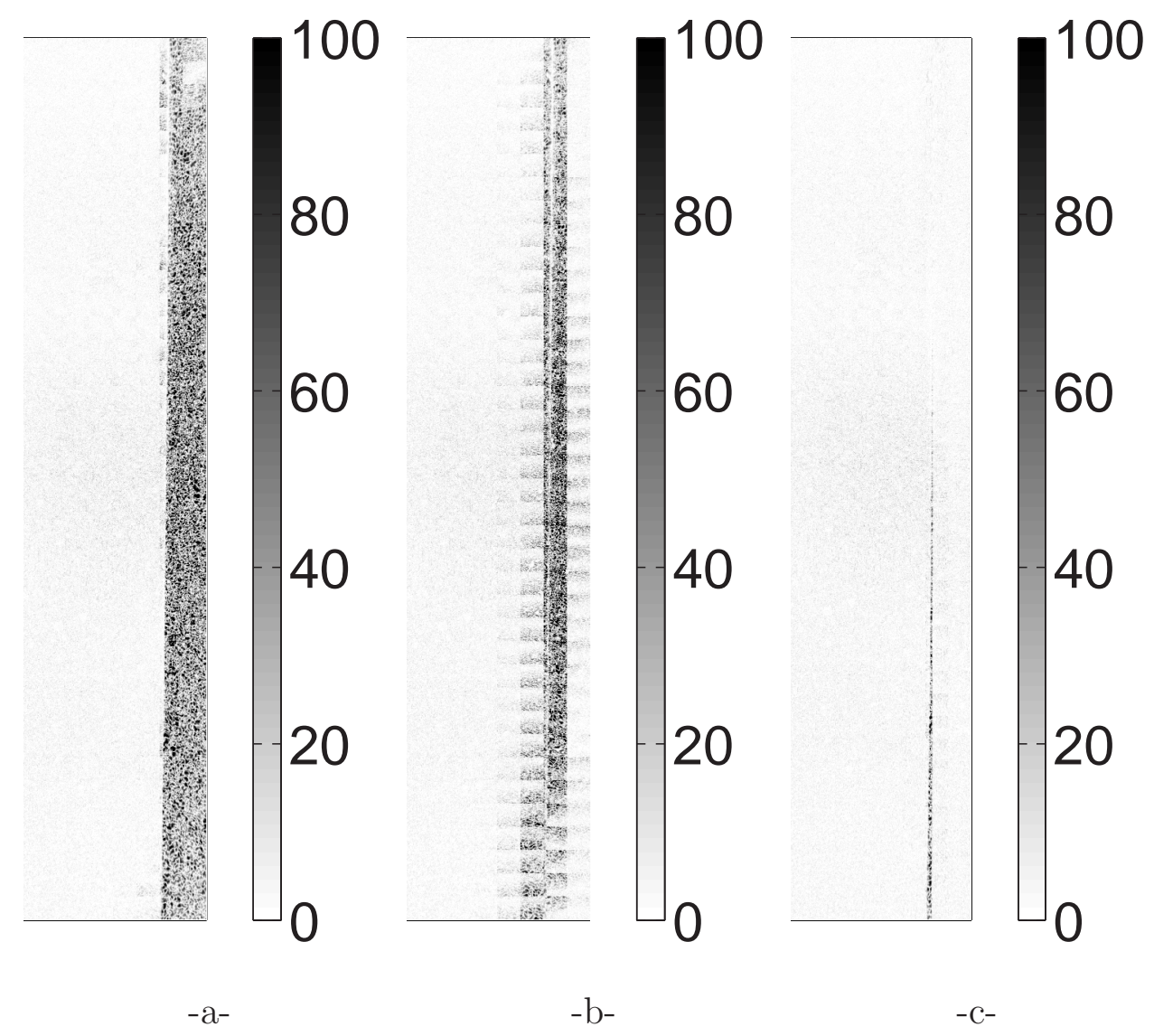

Fig. 12. Error map (in pixels) using 32-pixel elements with (a) Q4 interpolation on 1 grid,(b) Q4 interpolation on 5 grids, (c) discontinuous enrichment tangent to the interface on 5 grids 


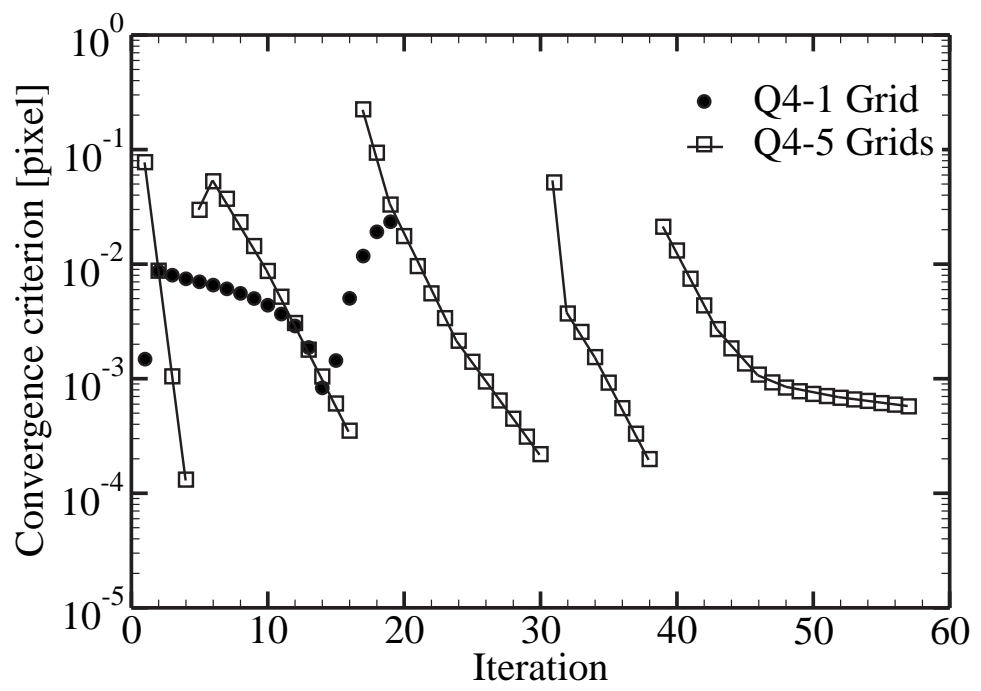

Fig. 13. Comparison of the convergence for analyses using 1 or 5 grids of 32-grain elements with a Q4P1 interpolation. The convergence criterion to reach is $10^{-4}$ pixel. Neither 1-grid nor 5-grid analyses allow for convergence at the true scale (grid 1)

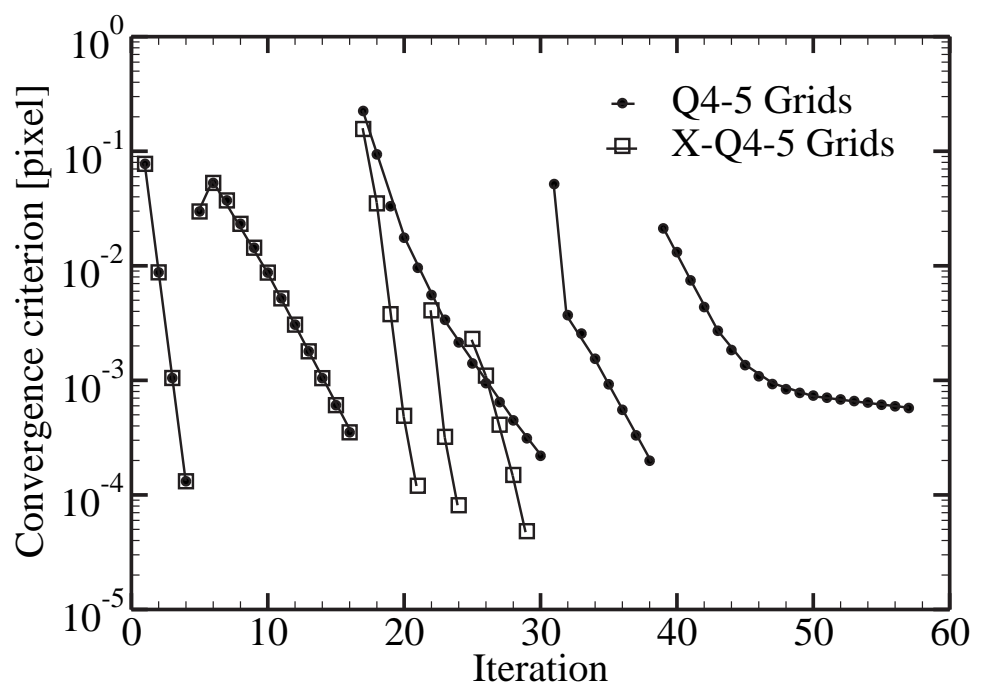

Fig. 14. Comparison of the convergence for analyses using 5 grids of 32-grain elements with a Q4P1 interpolation or an enrichment with discontinuous functions (the enrichment is introduced at grid 3). The convergence criterion to reach is $10^{-4}$ pixel. 


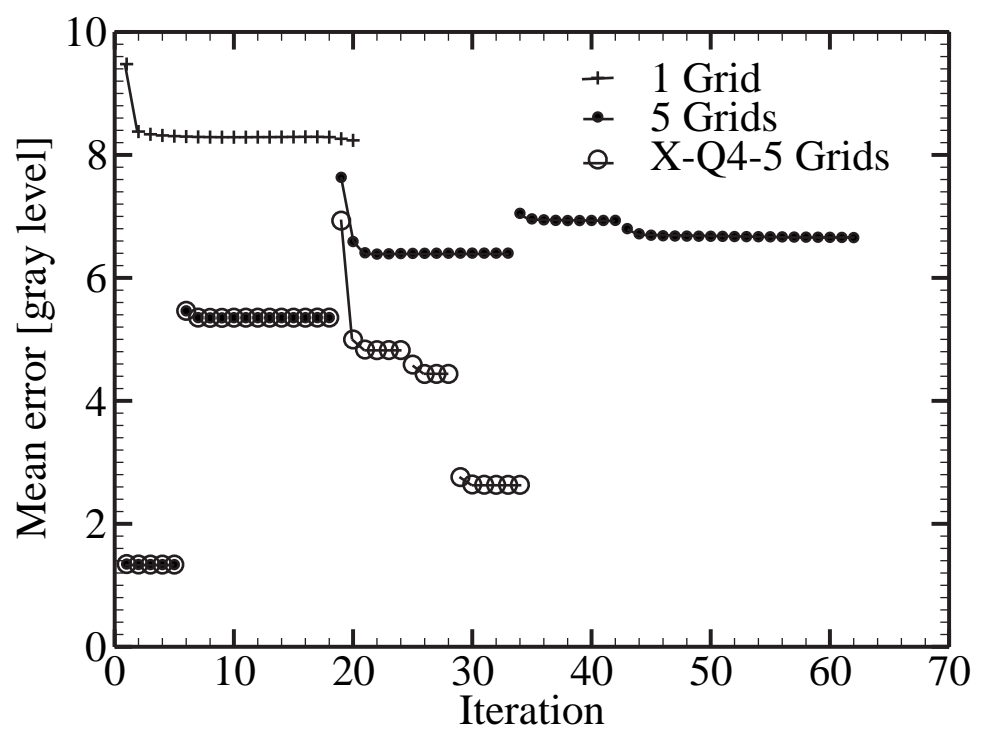

Fig. 15. Comparison of the mean error for analyses using 1 grid with a Q4 interpolation, 5 grids with a Q4 interpolation and 5 grids with an enrichment with discontinuous functions (the enrichment is introduced at grid 3). 32-grain elements are used for the analyses.
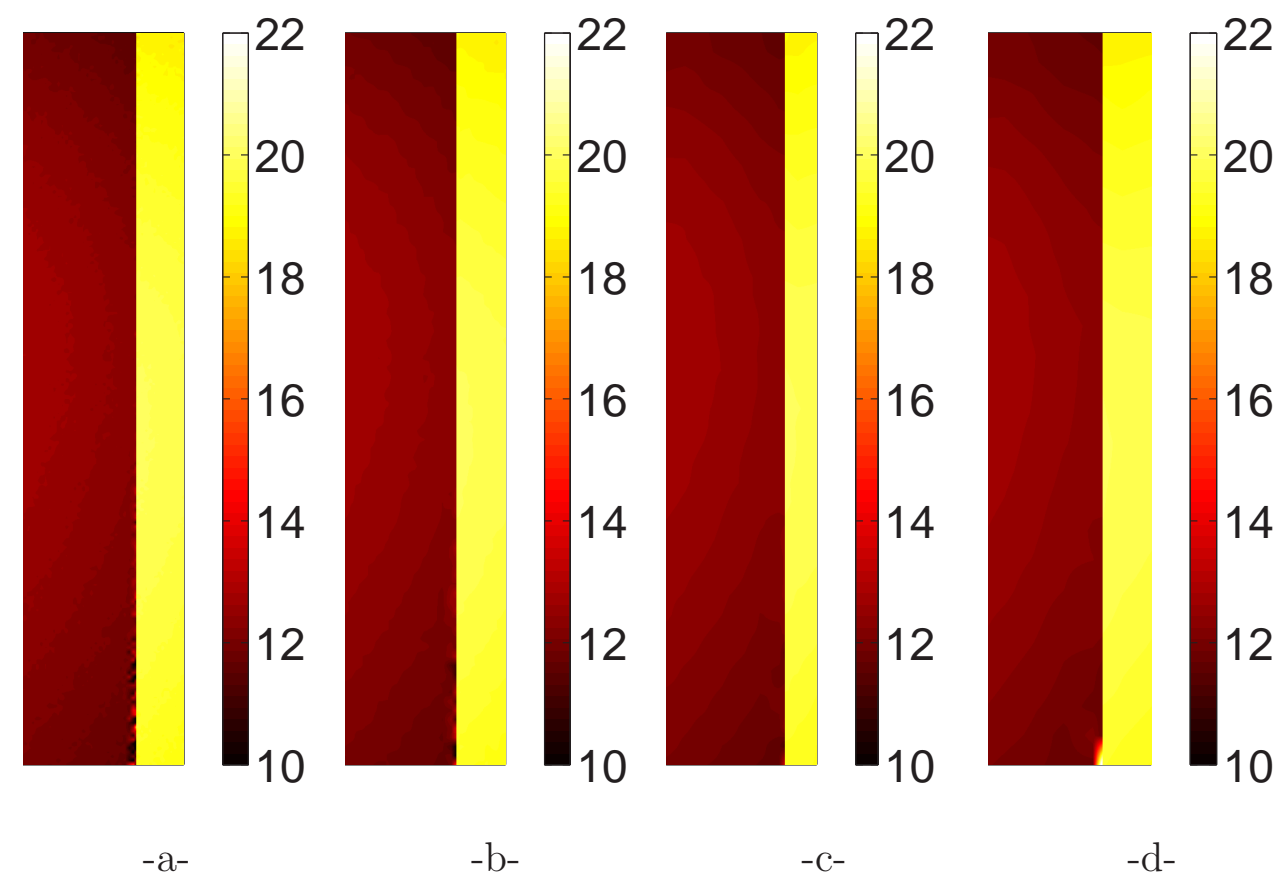

Fig. 16. Vertical displacement map (in pixels) using tangential enrichment for different discretizations: (a) 8-pixel elements, (b) 16-pixel elements, (c) 32-pixel elements, (d) 48-pixel elements 


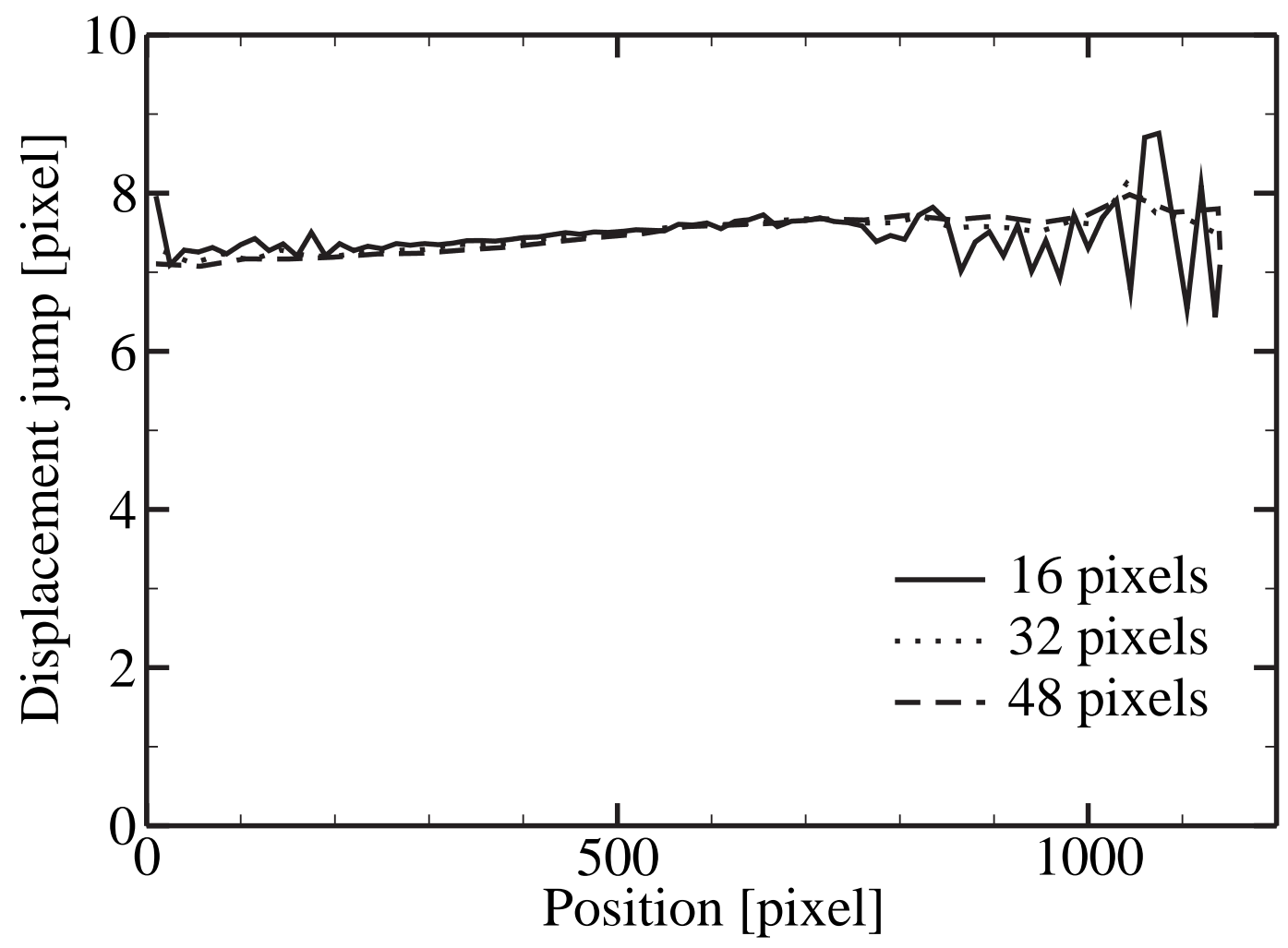

Fig. 17. Comparison of displacement jump profiles for different element sizes 


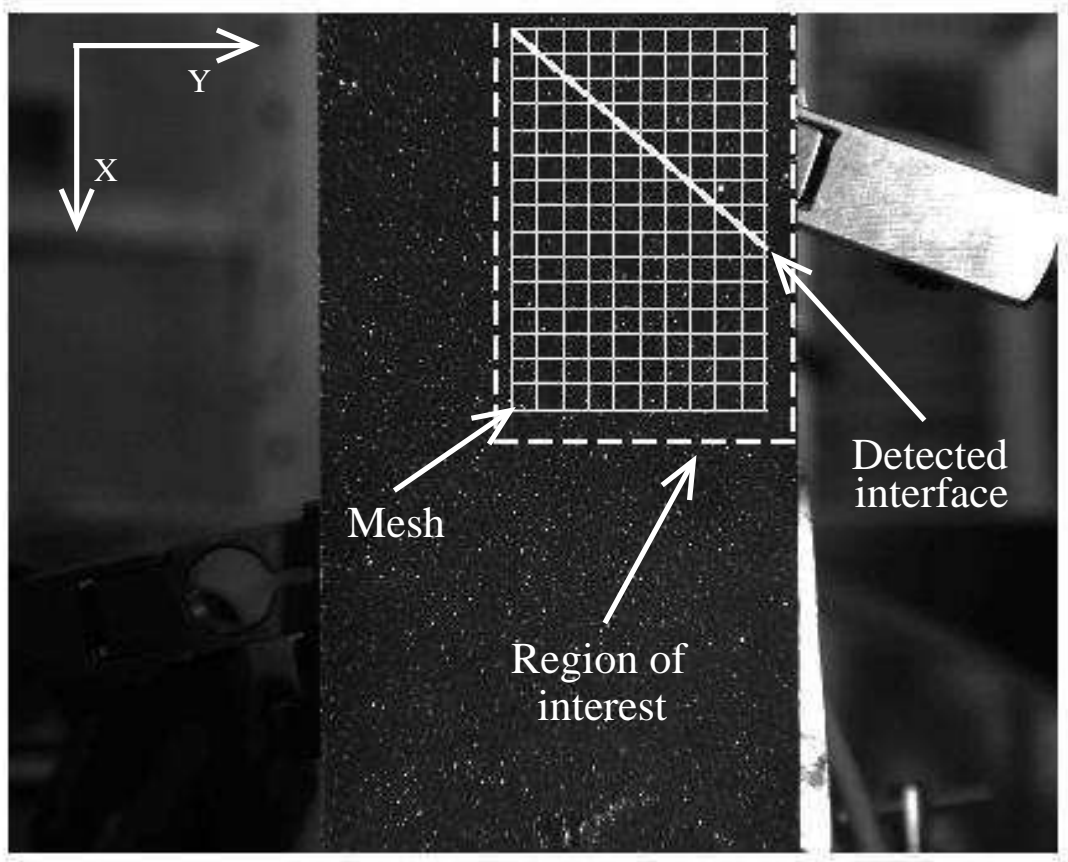

Fig. 18. Region of interest, mesh (grid 1) and detected shear band

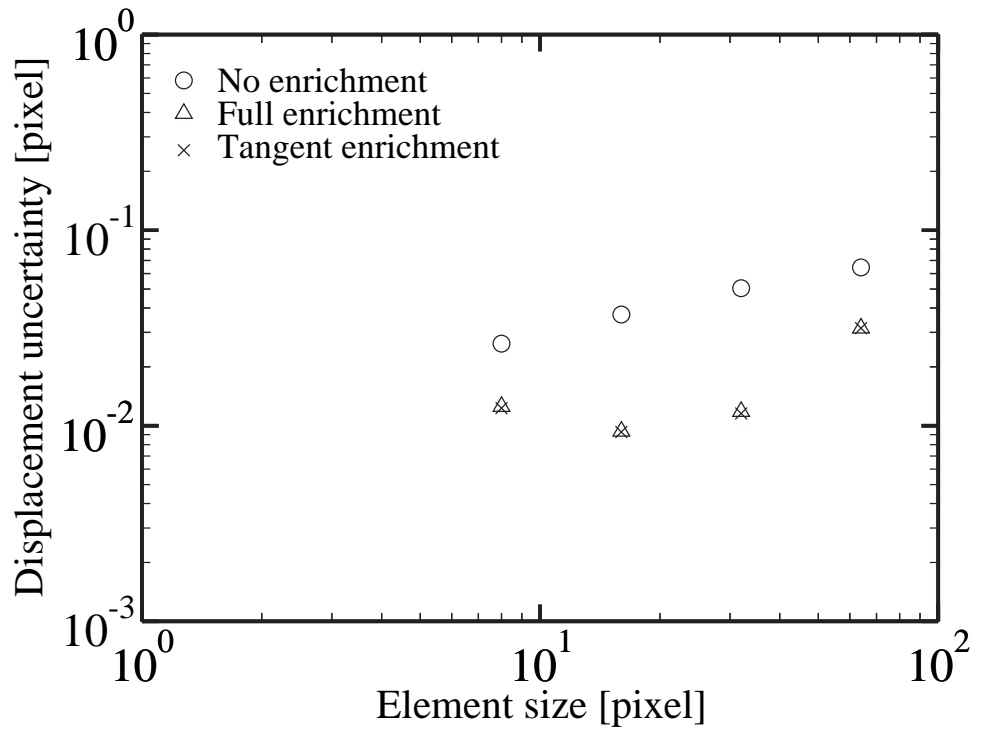

Fig. 19. Uncertainty of the displacement map for an artificial discontinuous displacement in the tangential direction. 


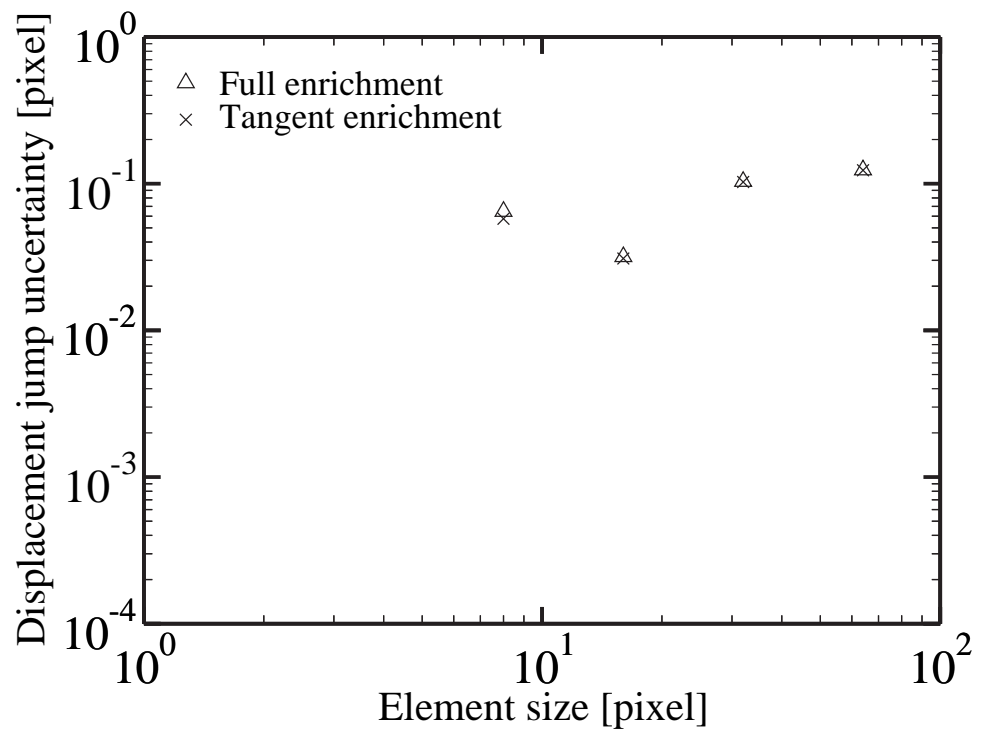

Fig. 20. Uncertainty of the displacement jump for an artificial discontinuous displacement in the tangential direction.

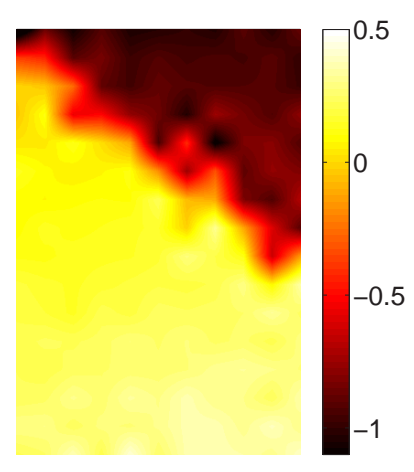

$-a-$

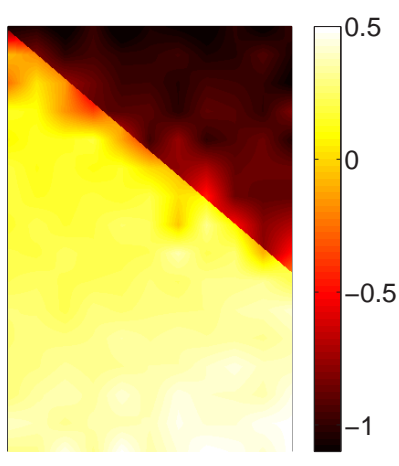

$-b-$

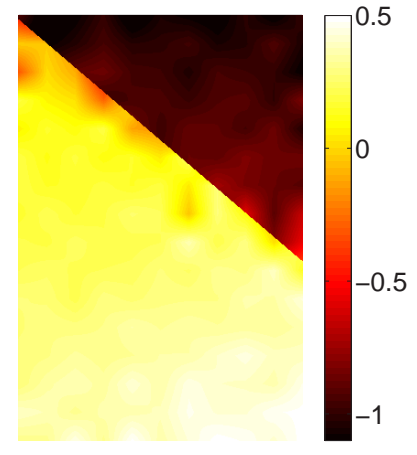

$-\mathrm{c}-$

Fig. 21. Vertical displacement map (in pixels) using 32-pixel elements with (a) Q4 interpolation, (b) discontinuous enrichment tangent to the band, (c) discontinuous enrichment tangent and normal to the band 


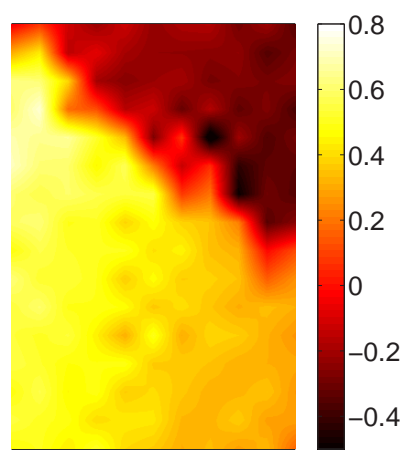

$-a-$

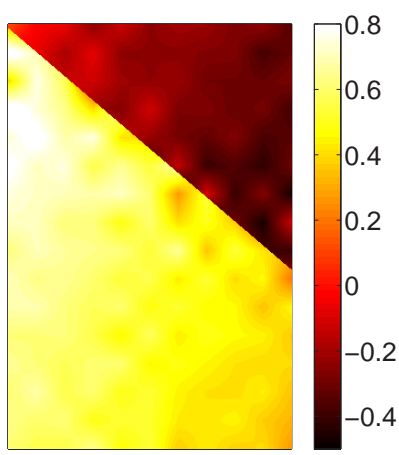

$-b-$

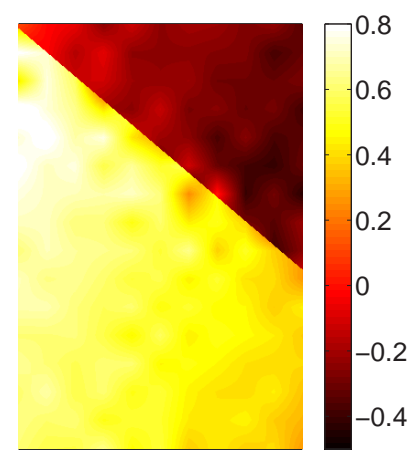

$-\mathrm{C}-$

Fig. 22. Horizontal displacement map (in pixels) using 32-pixel elements with (a) Q4 interpolation, (b) discontinuous enrichment tangent to the band, (c) discontinuous enrichment tangent and normal to the band 
Equivalent strain
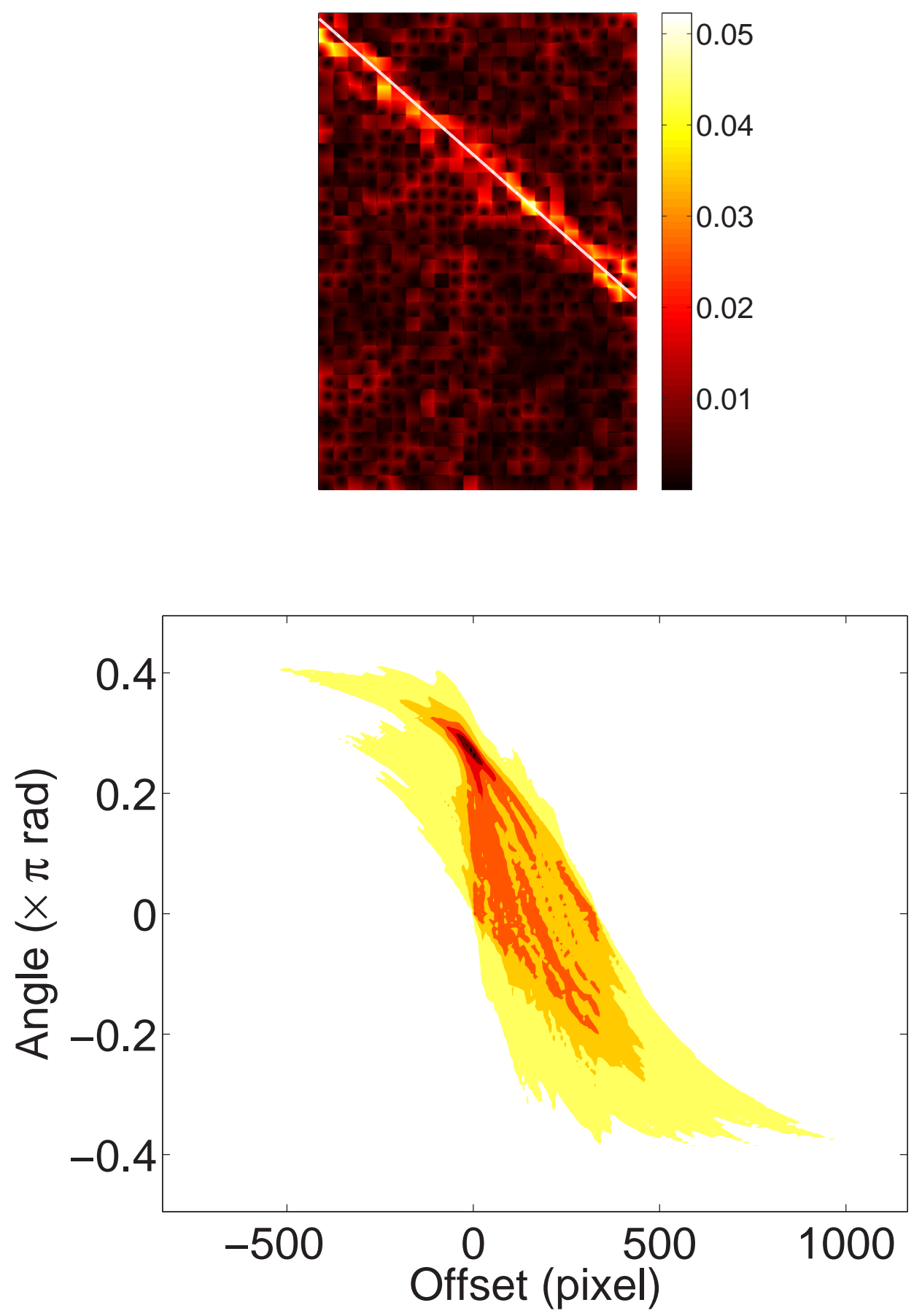

Fig. 23. Top: equivalent strain map; bottom: detection criterion 


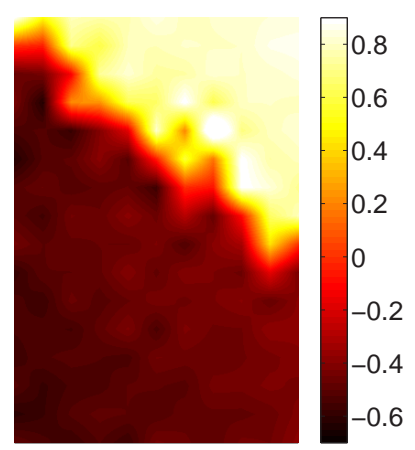

$-\mathrm{a}-$

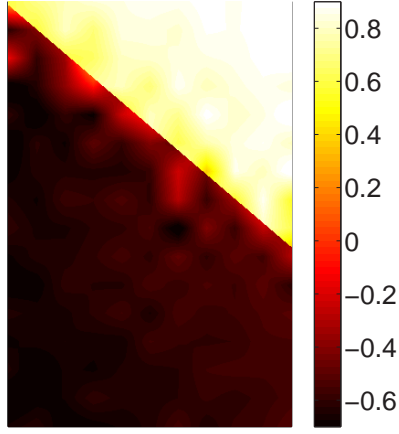

$-b-$

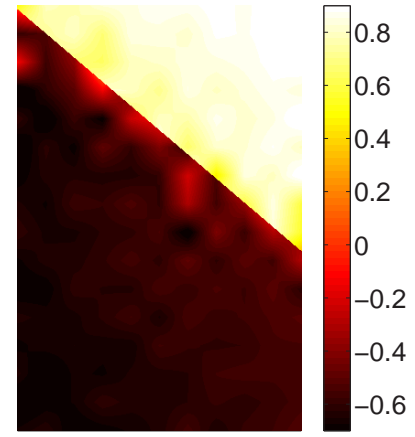

$-\mathrm{C}-$

Fig. 24. Tangent displacement map (in pixels) using 32-pixel elements with (a) Q4 interpolation, (b) discontinuous enrichment tangent to the band, (c) discontinuous enrichment tangent and normal to the band

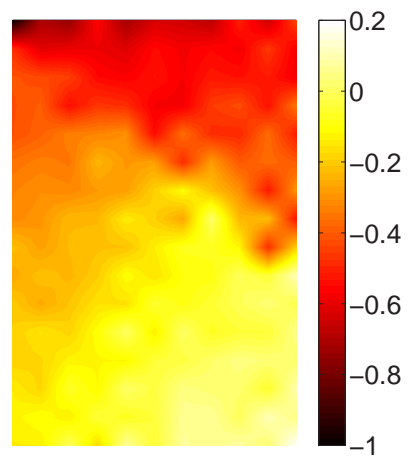

$-a-$
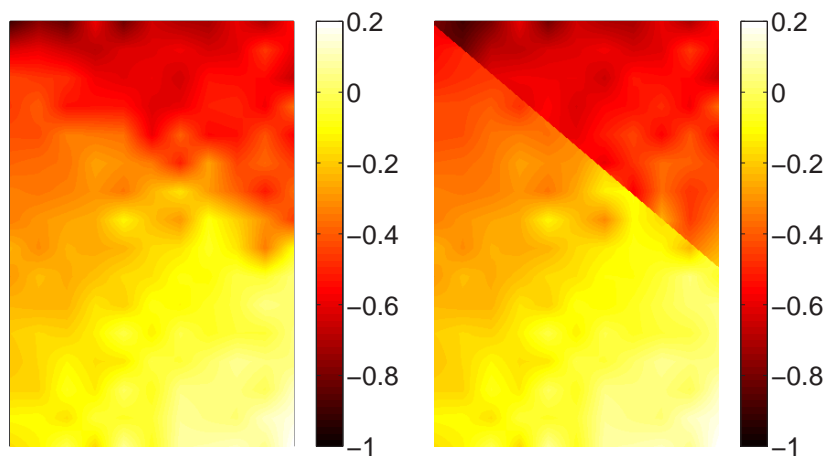

$-\mathrm{b}-$
$-\mathrm{C}-$

Fig. 25. Normal displacement map (in pixels) using 32-pixel elements with (a) Q4 interpolation, (b) discontinuous enrichment tangent to the band, (c) discontinuous enrichment tangent and normal to the band 


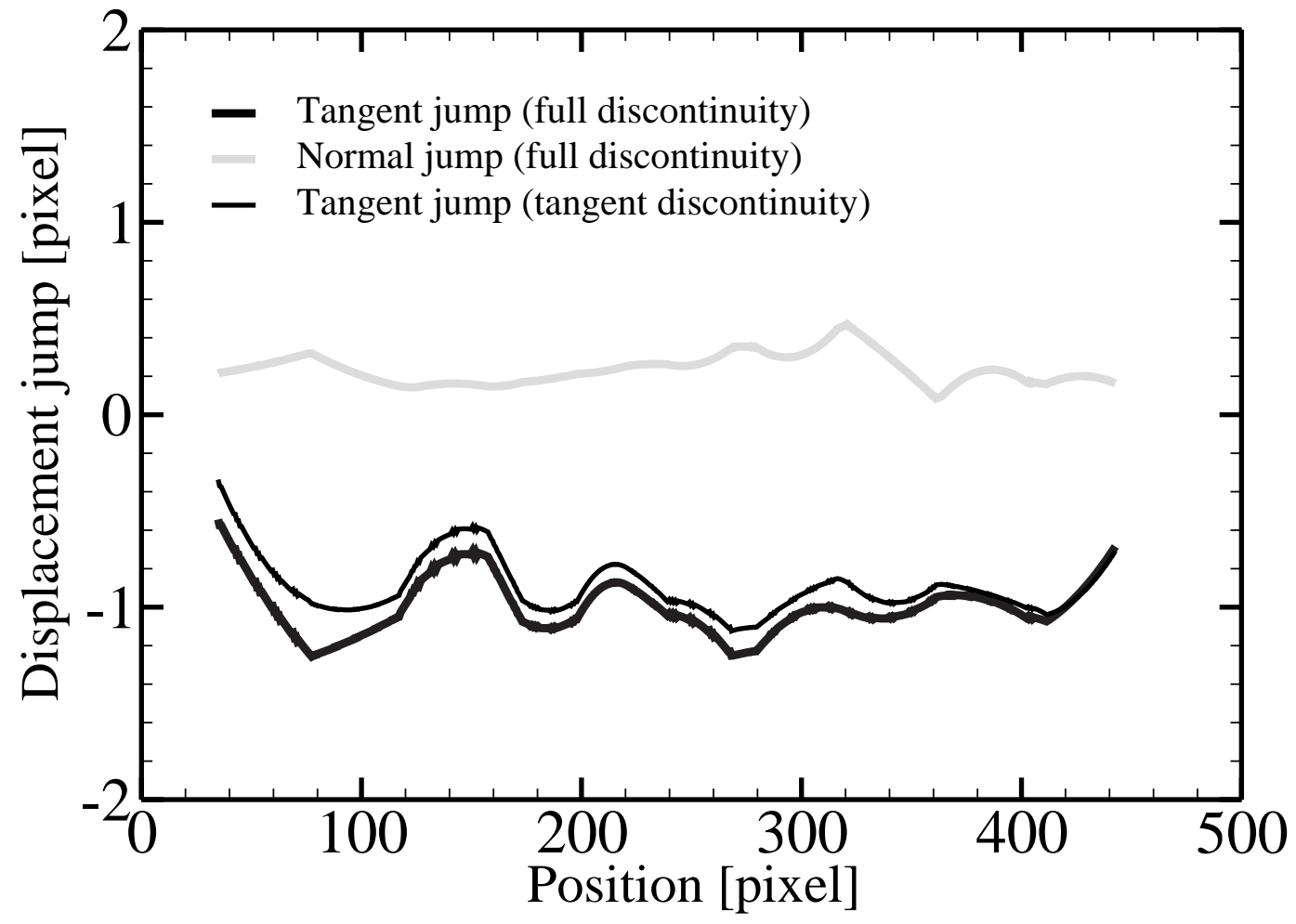

Fig. 26. Displacement jumps for 32-pixel elements with or without normal enrichment 


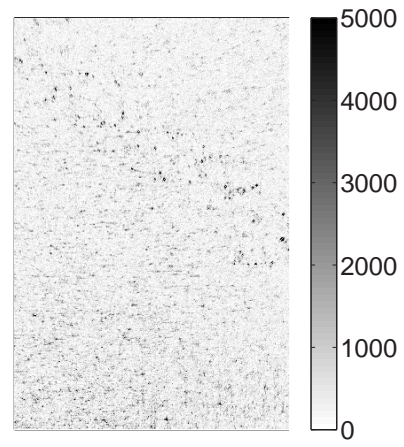

$-\mathrm{a}-$

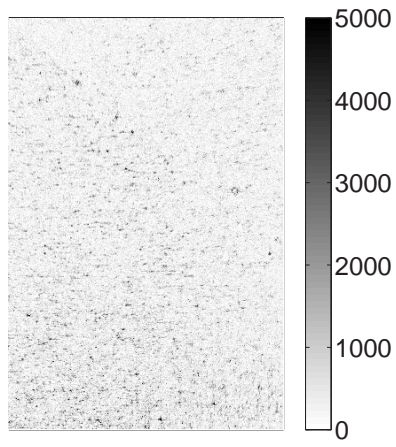

$-b-$

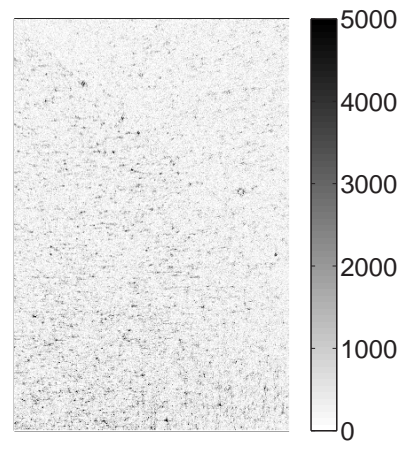

$-\mathrm{C}-$

Fig. 27. Error map (in gray levels) using 32-pixel elements with (a) Q4 interpolation, (b) discontinuous enrichment tangent to the band, (c) discontinuous enrichment tangent and normal to the band. The scale is saturated to 5000 gray levels in an initial range of $2^{16}$ gray levels 